\title{
Plasma biochemistry RIs and age effect in European Strigiformes
}

\author{
Susana Agusti Montolio ${ }^{1}$ | Rafaela Cuenca Valera ${ }^{1}$ | Santiago Lavin González ${ }^{1}$ | \\ Carolyn Cray $^{2}$ | Rafael Molina López ${ }^{3}$ | Emmanuel Serrano Ferron ${ }^{1,4}$ \\ Olga Nicolás Francisco ${ }^{5}$ | Ignasi Marco Sánchez ${ }^{1}$ | Encarna Casas-Díaz ${ }^{1}$
}

\author{
${ }^{1}$ Department of Animal Medicine and \\ Surgery, Universitat Autonoma de \\ Barcelona, Cerdanyola del Valles, Spain \\ ${ }^{2}$ Division of Comparative Pathology, \\ Department of Pathology, University of \\ Miami Miller School of Medicine, Miami, \\ FL, USA \\ ${ }^{3}$ Torreferrusa Wildlife Rehabilitation Center \\ (Catalan Wildlife Service, Forestal Catalana), \\ Santa Perpètua de Mogoda, Spain \\ ${ }^{4}$ Department of Biology \& CESAM, \\ Universidade de Aveiro (UA), Aveiro, Spain \\ ${ }^{5}$ Vallcalent Wildlife Rehabilitation Center \\ (Catalan Wildlife Service, Forestal Catalana), \\ Lleida, Spain \\ Correspondence \\ S. Agusti Montolio, Department of animal \\ Medicine and Surgery, Faculty of Veterinary \\ Medicine, Travessera dels Turons, $\mathrm{s} / \mathrm{n}$, \\ 08193 Cerdanyola del Valles, Barcelona, \\ Spain \\ E-mail: ssusanaa77@yahoo.es
}

Background: Blood biochemistry and hematology are essential in the laboratory diagnosis of disease. In Strigiformes, little information regarding reference values and influence of different preanalytic factors is available, while age is known to have an effect on some biochemistry analytes, especially in early life characterized by a rapid growth rate and increase in body mass.

Objective: The objective of this study was to determine baseline data for 29 blood biochemistry variables in 5 species of Iberian Strigiformes assigned to different age classes.

Methods: Healthy nocturnal birds living in wildlife health centers of Catalonia, Northeastern Spain were assigned to different age classes (chicks, juveniles, adults where possible and available) and their blood was collected and analyzed for different standard biochemistry variables. Species included Tawny owls, Little owls, Longeared owls, Scop owls, and Barn owls.

Results: A total of 276 clinically healthy animals were sampled, including between 179 chicks, 52 juveniles, and 45 adults. The RIs of cholesterol, albumin, and osmolality were the only variables that did not show interspecific variability. Common trends between age class groups were observed in 4 species. Phosphorus and calcium concentrations and ALP activities decreased with increasing age, while concentrations of sodium and $\gamma$-globulins tended to increase in the Strigidae family. Differences were concentrated in chicks and no differences were observed between juveniles and adults in any variable.

Conclusions: Our results demonstrate the importance of defining biochemical RIs specific for certain age classes

KEYWORDS

Blood, owl, values

\section{INTRODUCTION}

In the Iberian Peninsula, the Strigiformes order is represented by 8 different species: Eagle owl (Bubo bubo), Tawny owl (Strix aluco), Long-eared owl (Asio otus), Short-eared owl (Asio flammeus), Little owl (Athene noctua), Scops owl (Otus scops), Tengmalm's owl
(Aegoluis funereus), and Barn owl (Tyto alba). The presence of these animals in urban environments exposes them to numerous threats, such as the poaching of young birds, road accidents, poisonings, and collisions with electric power structures making them common patients at wildlife recovery centers. ${ }^{1}$ Blood biochemistry and hematology analysis in birds are clinically important because it 
provides an evaluation of nutritional and health status, and it is the basis for the laboratory diagnosis of disease. Additionally, in wildlife conservation, blood biochemistry can help identifying the predominant health issues affecting natural populations. ${ }^{2}$ Although many studies on blood biochemistry in wild avian species have appeared in past years, few investigations have focused on the Strigiformes order. ${ }^{3-9}$

There is sparse information about reference values and what influence different physiologic states of the bird's life cycle have on these variables. Age is an important biologic factor that influences blood biochemistry, especially in early life characterized by a rapid growth rate and increase in body mass. Growth is a complex process involving numerous interactions between the endocrine regulatory system and other contributing factors such as genetic, nutritional, and environmental aspects. ${ }^{10}$ Biochemistry RI definition in other avian species has shown that the age of the animals significantly influences blood biochemistry variables. ${ }^{11-13}$

The aim of this study was to determine the RI of 29 biochemical analytes in different age groups in 5 species of Iberian Strigiformes from 2 wildlife rehabilitation centers of Catalonia in Northeastern Spain.

\section{MATERIAL AND METHODS}

\section{Birds}

The study was conducted between October 2010 until October 2012. Blood samples were collected from Tawny owls, Little owls, Scops owls, Long-eared owls, and Barn owls.

All birds except 13 of 29 Barn owl chicks born in captivity and included in a breeding program were from the wild and were temporarily under the care of the Torreferrusa (430260.70 E, 4597845.02 N) and Vallcalent (300397.05 E, 4610027.28 N) wildlife rehabilitation centers in Northeastern Spain. The age of the birds was determined according to the feather characteristics. Chicks were birds with downy plumage. Juveniles were birds without downy plumage, and that were born during the breeding season of that calendar year. Adults were birds with an unknown birth date that were born before the current calendar year. The sex was not determined as in these species genitals are not sufficiently pronounced to allow accurate sex determination.

Upon admission, chicks, juveniles, and adults were placed in individual cages located indoors. Healthy birds were moved to the outdoor aviaries where they were freely fed. Aviary flight rooms varied from $3 \times 3 \times 2$ to $10 \times 4 \times 3 \mathrm{~m}$. The top and sides were covered with wire netting, and weather protected sites and wooden perches were included. Owls of the same species were housed in the same flight room until release.

The diet of the birds included rats, mice, quail, and one-day-old chickens. Chicks were hand-fed shredded whole rat meat until the bird could self-feed and forage.

The animals were selected for the study according to health status, which included information obtained from the clinical history, the physical examination, and the results of laboratory tests including hematology. Any animal with signs of disease, unhealed injuries, anorexia, or weight loss was excluded from the study.

\section{Blood sample collection}

Blood sampling was always performed during the hours of 9 AM and $11 \mathrm{AM}$ and at least 8-12 $\mathrm{h}$ after the last feeding to reduce diurnal and postprandial blood analyte variation, respectively.

Chicks were hand-captured, and juveniles and adults were captured with nets. Birds were physically restrained, and blood was collected from the right jugular vein using disposable $1 \mathrm{~mL}$ plastic syringes (Omnifix-F; B. Braun, Melsungen, Germany) with 25-gauge needles (Sterican; B. Braun). Blood was placed in microtainer tubes (Becton-Dickinson and Co., Franklin Lakes, NY, USA) containing lithium heparin $(1.8 \mathrm{mg} / \mathrm{mL}$ blood) as an anticoagulant. Within $2 \mathrm{~h}$ after sampling, plasma was obtained by centrifuging (Rotofix 32A; Andreas Hettich GmbH \& Co., Tuttlingen, Germany) the blood (148g for $10 \mathrm{~min}$ ) and was immediately frozen at $-70^{\circ} \mathrm{C}$.

\section{Laboratory analyses}

Plasma specimens were used for biochemical analyses on an Olympus AU400 analyzer (Olympus, Mainz, Germany) including ion-selective electrodes. The following analytes were measured: AST, CK, uric acid, glucose, urea, triglycerides, glutamate dehydrogenase (GLDH), ALP, total bile acids, ALT, calcium, phosphorus, amylase, GGT, creatinine, cholesterol, magnesium, sodium, potassium, and chloride. All reagents were obtained from the analyzer manufacturer (Olympus System Reagent; Olympus, Dublin, Ireland) and the test principles are listed in Table 1.

Plasma osmolality was determined by the equation $2 \times\left(\mathrm{Na}^{+}+\mathrm{K}^{+}\right)+$(uric acid concentration/16.8) + (glucose concentration/18). ${ }^{14}$ Fibrinogen was determined by the heat precipitation method. ${ }^{15}$

Plasma protein electrophoresis was performed using cellulose acetate membranes (Cellogel electrophoresis Co., Milan, Italy) and a $0.04 \mathrm{M}$ sodium veronal buffer ( $\mathrm{pH}$ 8.6; ATOM, Barcelona, Spain) in an electrophoresis chamber (BioSystems Reagents \& Instruments, Barcelona, Spain) connected to an electrophoresis power supply BTS-100 (BioSystems Reagents \& Instruments). The electrophoretic separation was run for $35 \mathrm{~min}$ at $200 \mathrm{~V}$ at room temperature. The membranes were stained with a solution of amido black (Cellogel electrophoresis, Milan, Italy). Electrophoretograms were scanned on a BioSystems BTS-245 densitometer (BioSystems Reagents \& Instruments) at $570 \mathrm{~nm}$. The fractions determined were prealbumin, albumin, $\alpha$-globulins ( $\alpha-1$ and $\alpha-2$ were considered as a single fraction), $\beta$-globulins, and $\gamma$-globulins.

\section{Statistical analyses}

Descriptive statistics (mean, median, standard deviation, minimum, and maximum) and $\mathrm{RI}$ with a $90 \%$ confidence interval (CI) for the upper and lower limits were performed using the freeware program 
TAB LE 1. Methods used to measure biochemistry analytes in Tawny Owl, Little Owl, Scops Owl, Long-eared Owl, and Barn Owl.

\begin{tabular}{|c|c|}
\hline Analyte & Method \\
\hline AST & $\begin{array}{l}\text { Enzymatic and colorimetric method without addition of pyridoxal phosphate, tris (hydroxymethyl) } \\
\text { aminomethane buffer. Aspartate concentration }>100 \mathrm{Mm} \text {. }\end{array}$ \\
\hline CK & $\begin{array}{l}\text { Enzymatic and colorimetric method } \\
\text { UV method with } \mathrm{N} \text {-acetylcysteine activator. }\end{array}$ \\
\hline Uric Acid & Uricase/PAP \\
\hline Glucose & Hexokinase \\
\hline Urea & Urease with glutamate dehydrogenase \\
\hline Triglycerides & Enzymatic of glycerol phosphate oxidase \\
\hline Glutamate dehydrogenase & $\begin{array}{l}\text { Recommended by the Deutsche Gesellschaft für Klinische Chemie. } \\
\text { ( } \alpha \text {-oxoglutarate }+\mathrm{NADH}+\mathrm{NH}_{4} \text { 口 glutamate }+\mathrm{NAD}^{+}+\mathrm{H}_{2} \mathrm{O} \text { ). }\end{array}$ \\
\hline ALP & Recommended by the IFCC with nitrophenyl phosphate with buffer AMP. \\
\hline Total Bile Acids & Ammonia Enzymatic UV, Randox, Ardmore, United Kingdom. \\
\hline ALT & $\begin{array}{l}\text { Enzymatic and colorimetric method, without addition of pyridoxal phosphate, tris (hydroxymethyl) } \\
\text { aminomethane buffer, alanine concentration }>225 \mathrm{Mm}\end{array}$ \\
\hline Calcium & Arsenazo III \\
\hline Phosphorus & Molybdate \\
\hline Amylase & Colorimetric method, with addition of ethylidene-G $-\mathrm{PNP}$. \\
\hline GGT & Recommended by the IFCC (with gamma-glutamyl-3-carboxy-4-nitroanilide with concentration $>4 \mathrm{mmol} / \mathrm{L}$ ). \\
\hline Creatinine & Jaffé. \\
\hline Cholesterol & Cholesterol esterase enzymatic/POD. \\
\hline Magnesium & Colorimetric with direct blue xilidyl. \\
\hline Sodium, potassium, and chloride & Ion-specific electrode. Diluted. \\
\hline
\end{tabular}

TAB LE 2. Descriptive statistics and Rls for Cholesterol, Osmolarity, and Albumin in Tawny owl, Little owl, Long-eared owl, Scops owl, and Barn owl.

\begin{tabular}{|c|c|c|c|c|c|c|c|c|c|c|c|}
\hline \multirow[b]{2}{*}{ Variable } & \multirow[b]{2}{*}{ Units } & \multicolumn{5}{|c|}{ Descriptive Statistics } & \multicolumn{2}{|l|}{ RI with $90 \% \mathrm{CI}$} & \multicolumn{3}{|c|}{ Reference Value Advisor } \\
\hline & & Mean & Median & SD & Minimum & Maximum & Lower Limit (RI) & Upper Limit (RI) & $n$ & Distribution & Method \\
\hline Cholesterol & $\mathrm{mmol} / \mathrm{L}$ & 8.78 & 8.54 & 1.81 & 4.60 & 13.22 & $5.01(4.64-5.33)$ & $\begin{array}{l}12.23 \\
(11.80-12.72)\end{array}$ & 207 & NG & RT \\
\hline Osmolarity & mosm $/ \mathrm{Kg}$ & 324.52 & 324.11 & 7.11 & 307.73 & 341.78 & $\begin{array}{l}310.35 \\
(309.08-311.64)\end{array}$ & $\begin{array}{l}338.5 \\
(337.14-340.08)\end{array}$ & 209 & G & $\mathrm{R}$ \\
\hline Albumin & $\mathrm{g} / \mathrm{L}$ & 16.38 & 16.35 & 2.09 & 11.10 & 21.40 & $12.26(11.91-12.62)$ & $\begin{array}{l}20.05 \\
(20.12-20.86)\end{array}$ & 242 & G & $\mathrm{R}$ \\
\hline
\end{tabular}

$n$ indicates sample size; G, Gaussian distribution; NG, non-Gaussian distribution; R, robust method; RT, robust method after Box-Cox transformation.

Reference Value Advisor ${ }^{16}$ (National Veterinary School, Toulouse, France; available at: http://www.biostat.envt.fr/spip.php) in accordance with the American Society of Veterinary Clinical Pathology (ASVCP) guidelines. ${ }^{17,18}$

To explore age variation in the Tawny owl, Little owl, Scops owl, and Barn owl, we eliminated outliers as defined by a box plot and by values 1.5 times the interquartile range (IQR) above the upper quartile or below the lower quartile.

Normality of the data was tested with the Kolmogorov-Smirnov test, and homogeneity of variances was tested using Levene's test. Statistical comparisons between the different ages were made using the Kruskal-Wallis test for nonparametric data and the one-way ANOVA for parametric data. A $P<.05$ was considered significant. A 2-tailed $t$-test was employed to explore the differences of the means between the different ages. The $R$ project $(R$ version 3.2.1 development core team, 2015. R foundation for statistical computing, Vienna, Austria, http://www.R-project.org/) was used for statistical analyses.

\section{RESULTS}

Seventy-six Tawny owls (48 chicks; 15 juveniles; 13 adults), 76 Little owls (48 chicks; 15 juveniles; 13 adults), 74 Scops owls (46 chicks; 14 juveniles; 14 adults), 8 Long-eared owls (8 chicks), and 42 Barn owls (29 chicks; 8 juveniles; 5 adults) were included. No adults or juveniles of Long-eared owl were sampled. All plasma samples were 


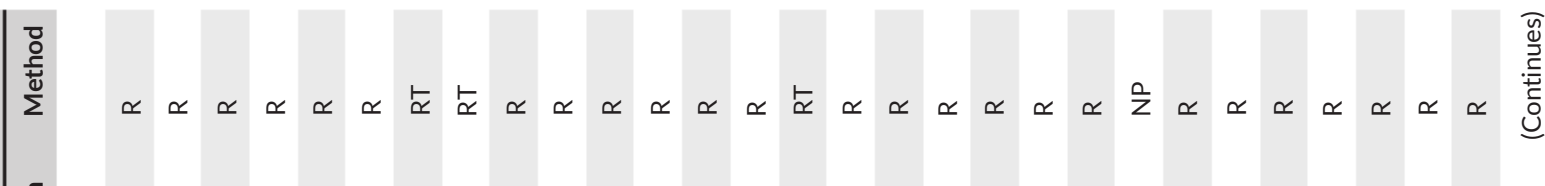

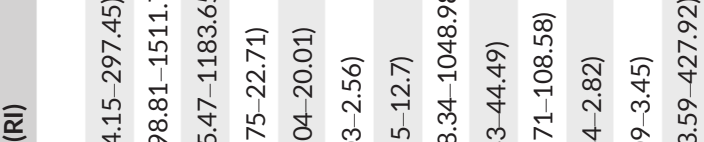

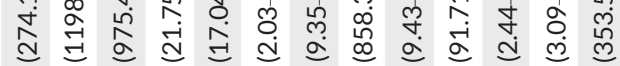

K a d ने +

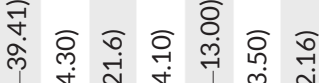

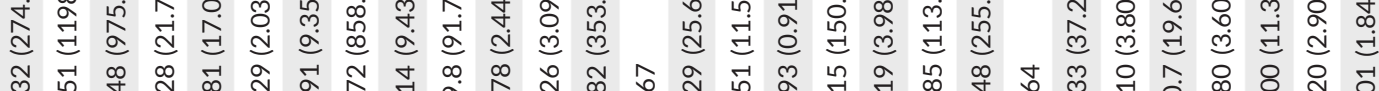

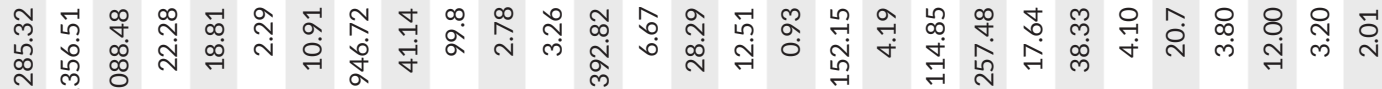

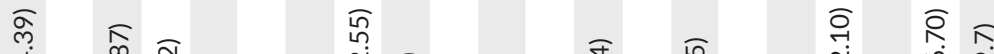

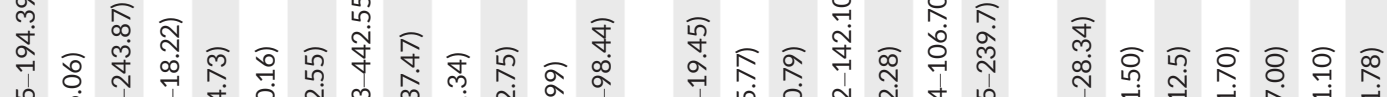
น ग

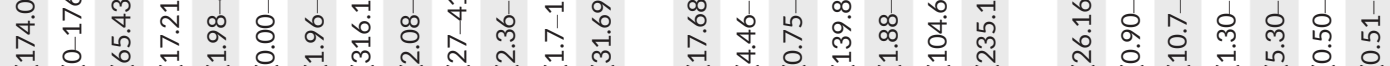

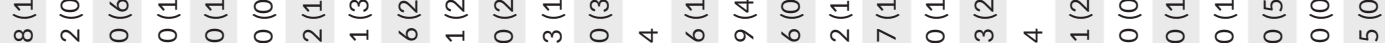

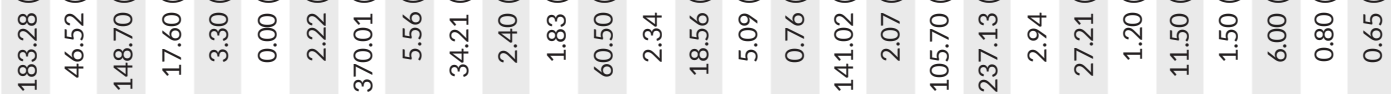

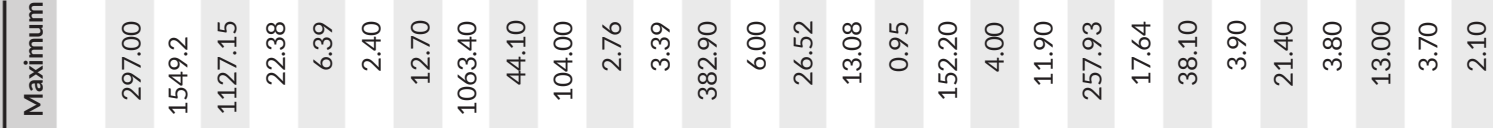

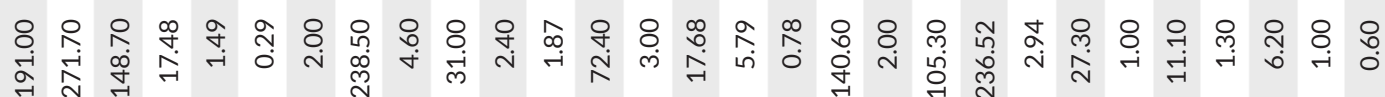

先 每

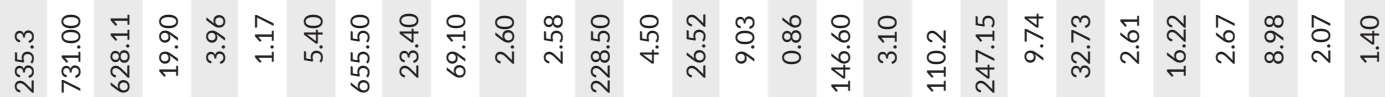

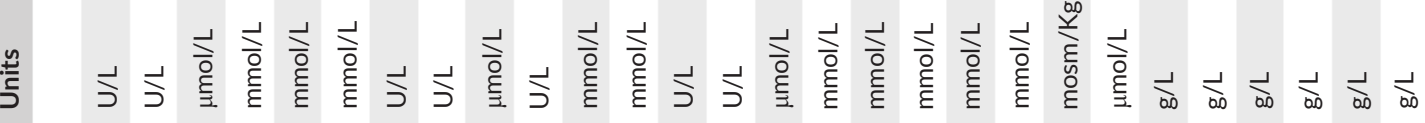




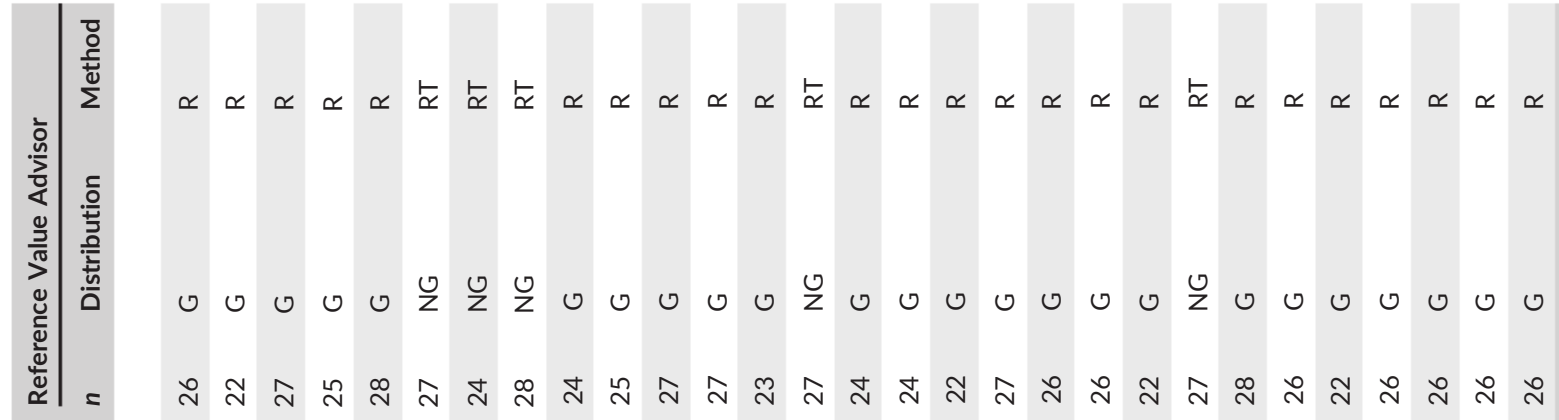

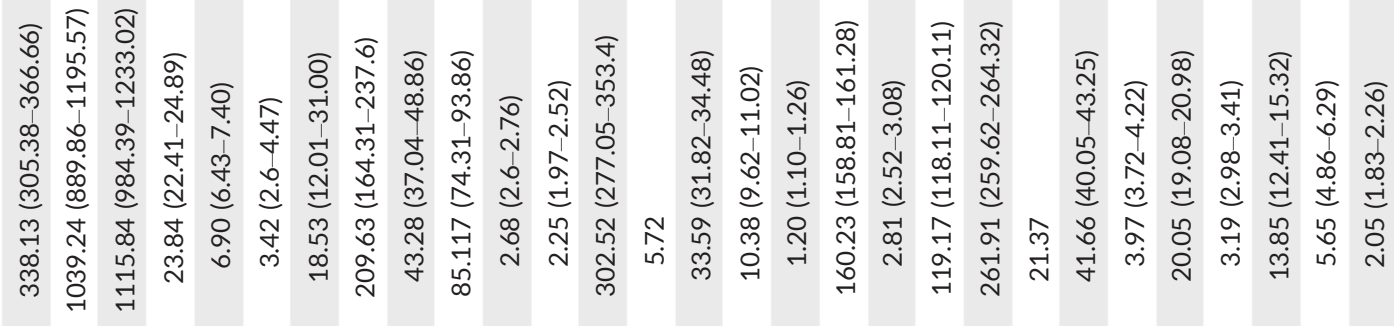

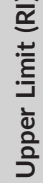

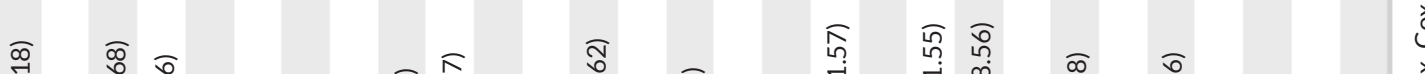

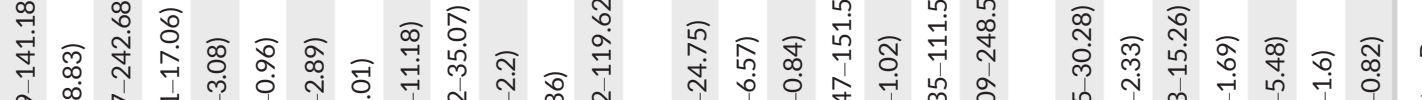

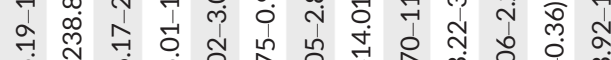
点

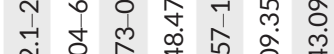

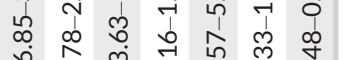

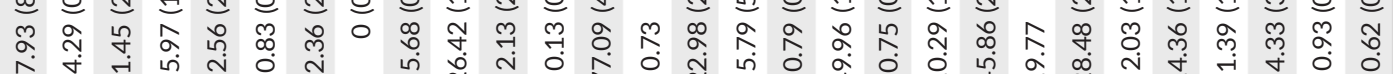

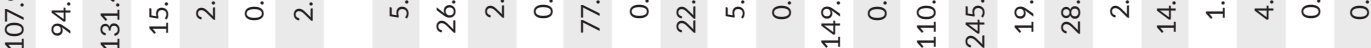

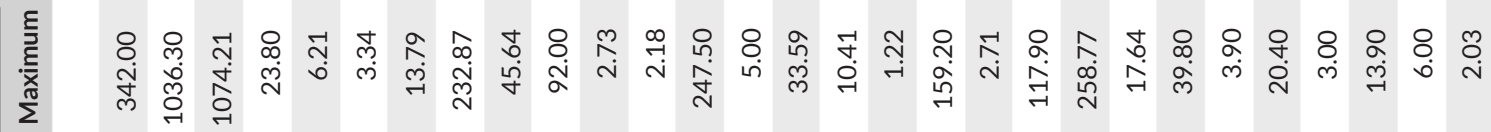

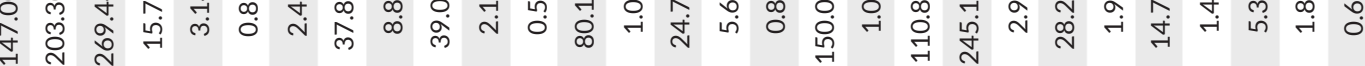

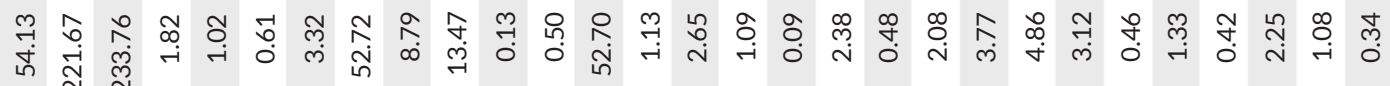

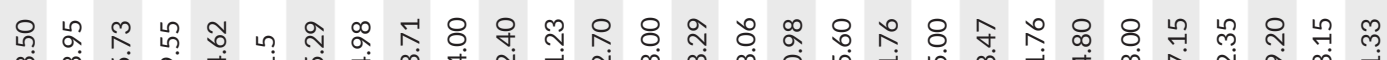

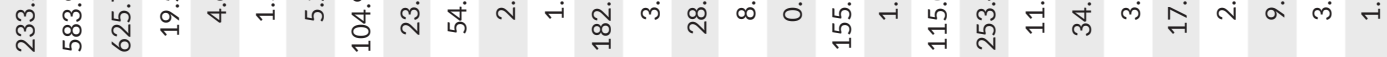

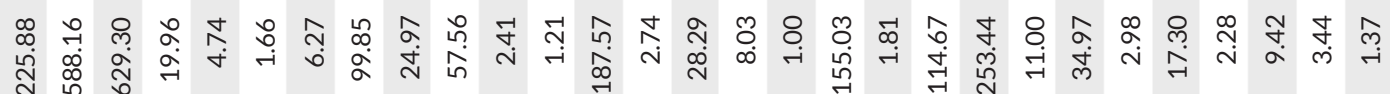

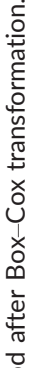

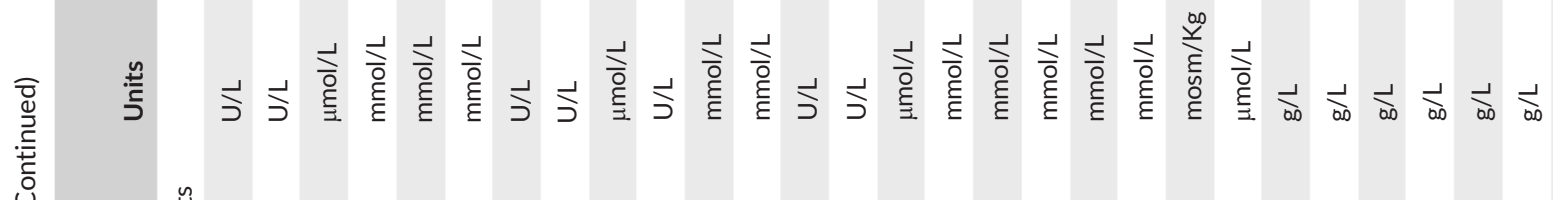

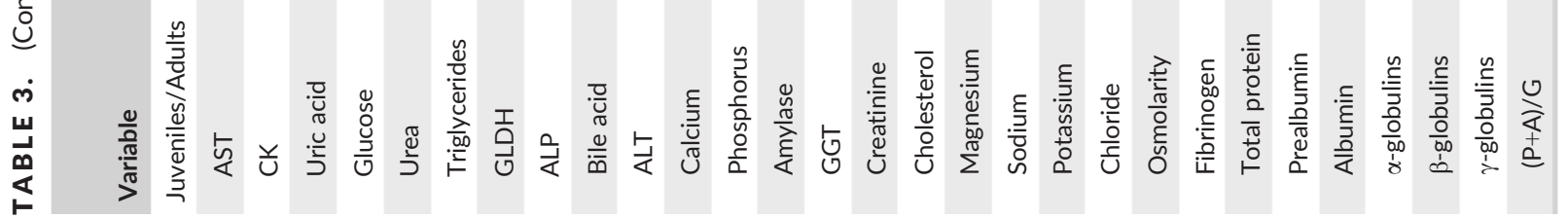




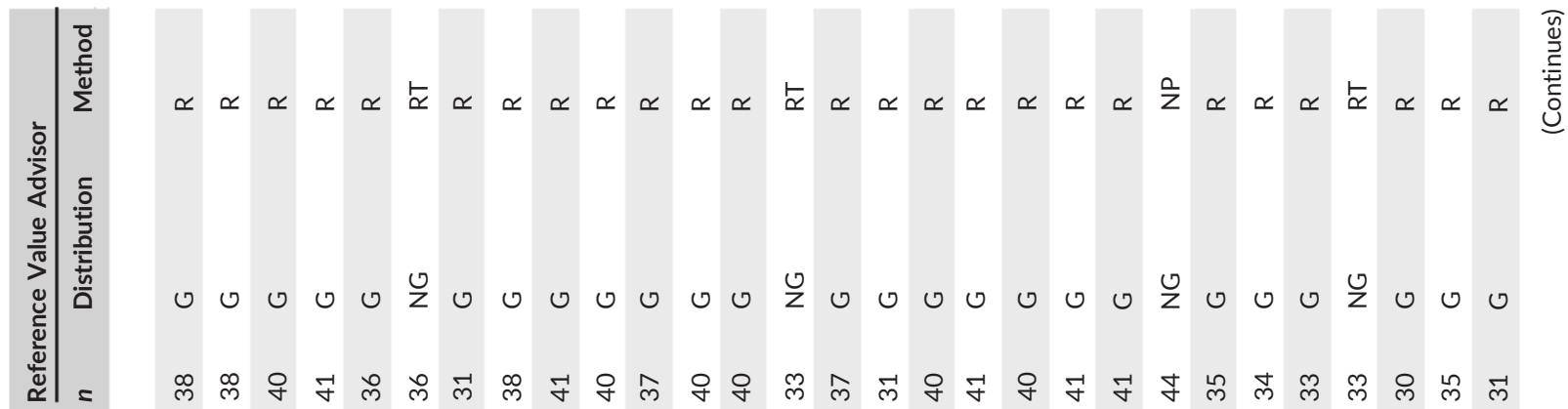

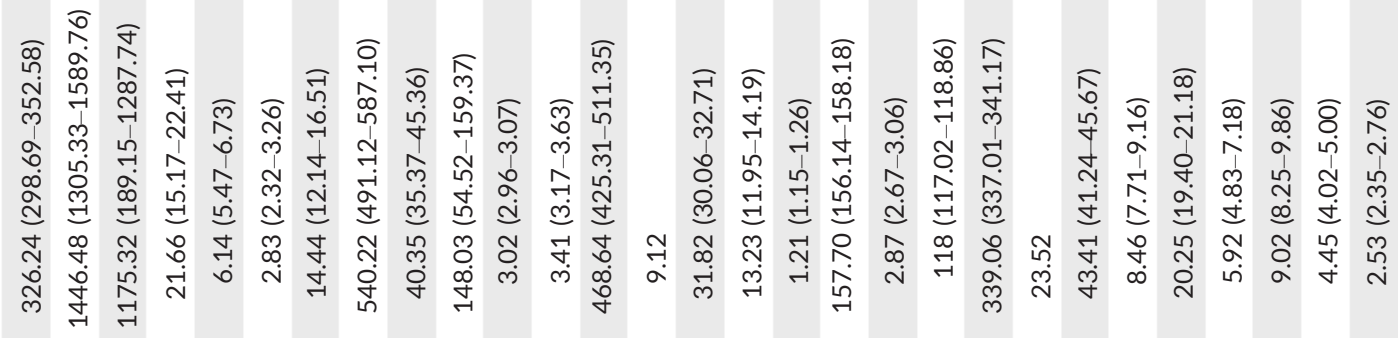

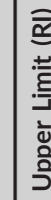

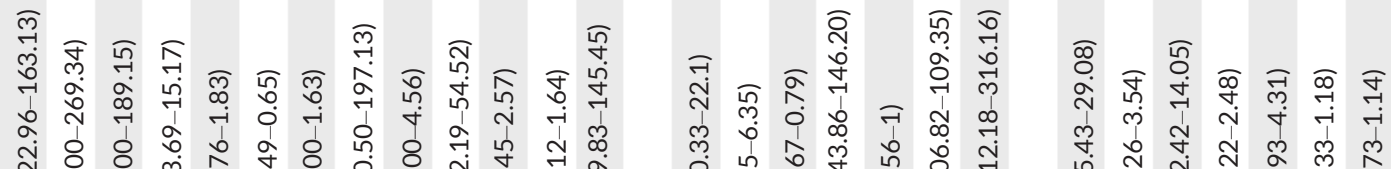

每

我

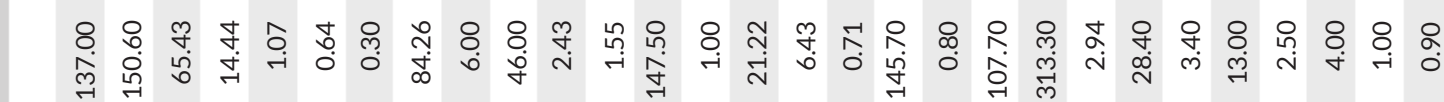

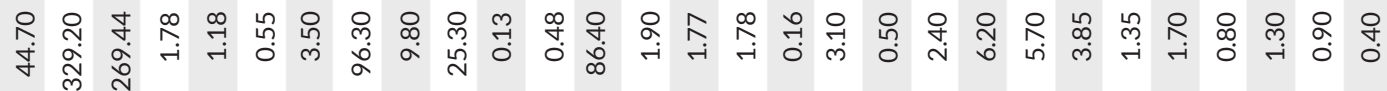

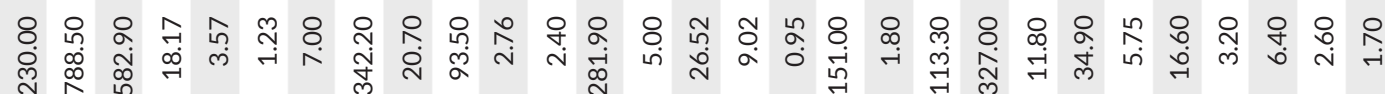

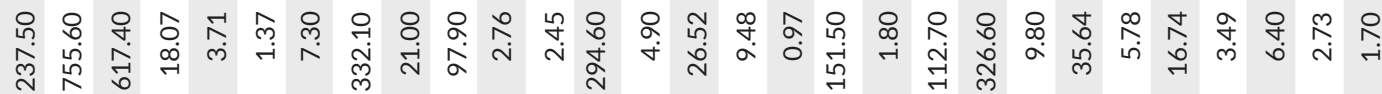

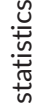

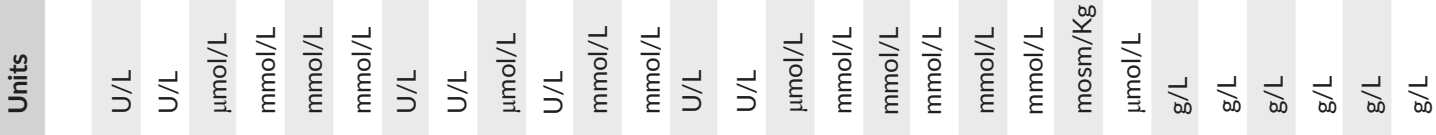




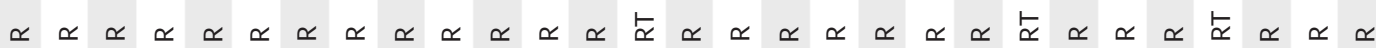

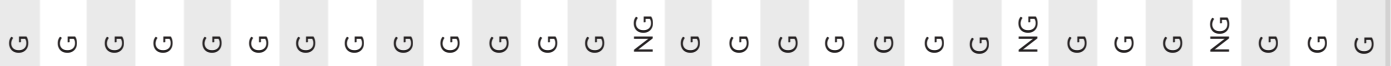

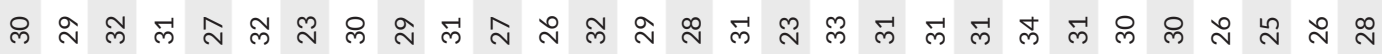

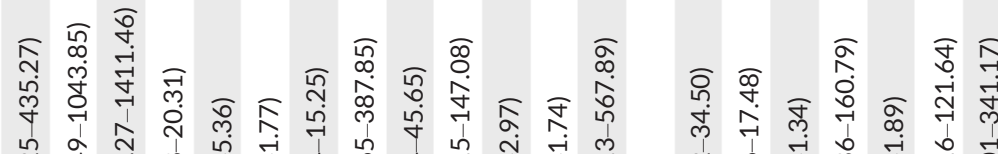

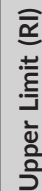

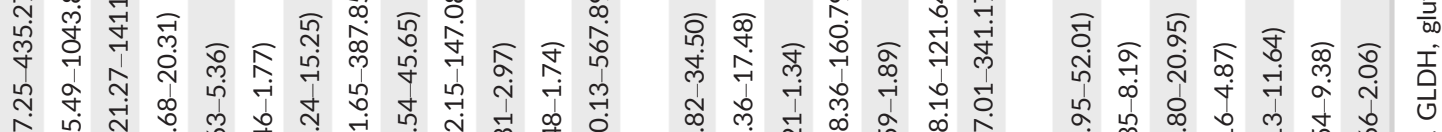

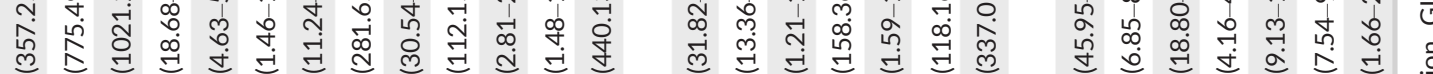

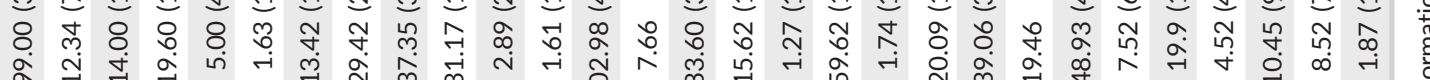

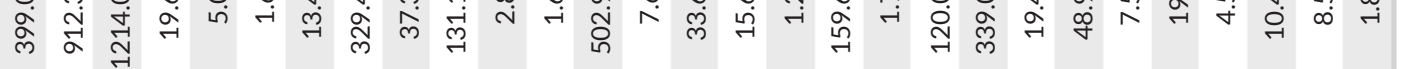

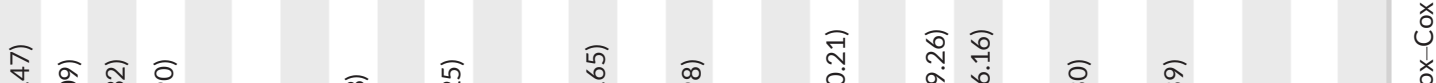

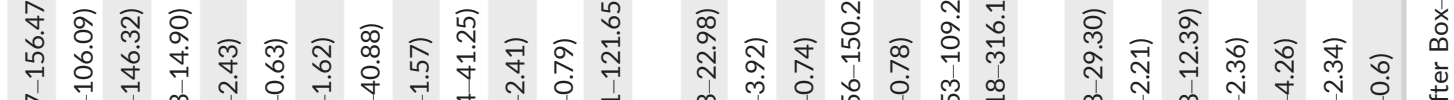

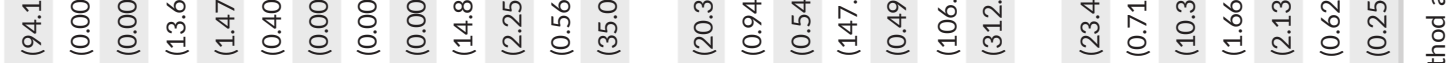

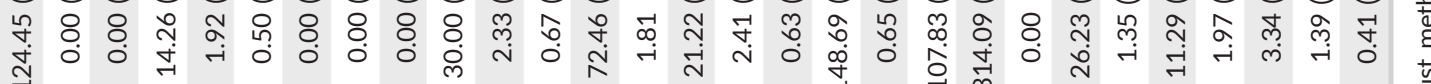

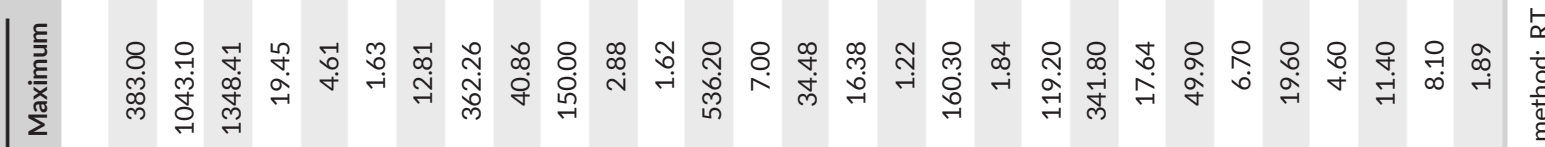

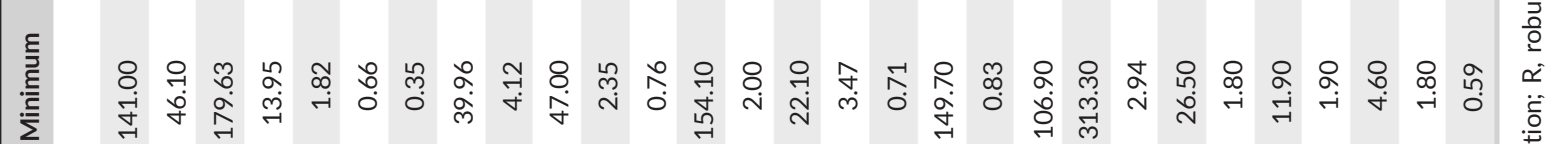

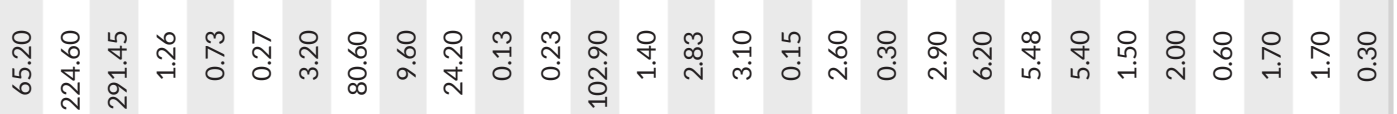

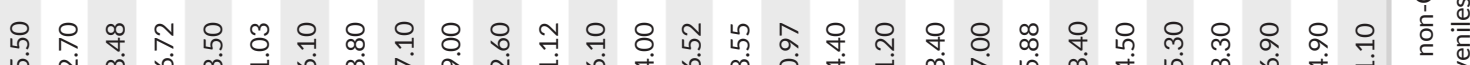
岗

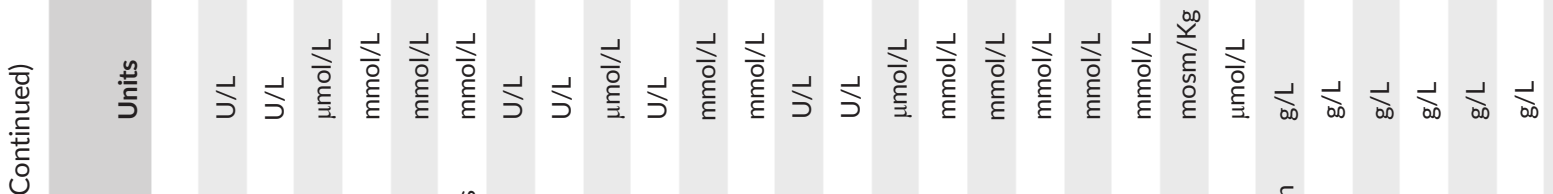




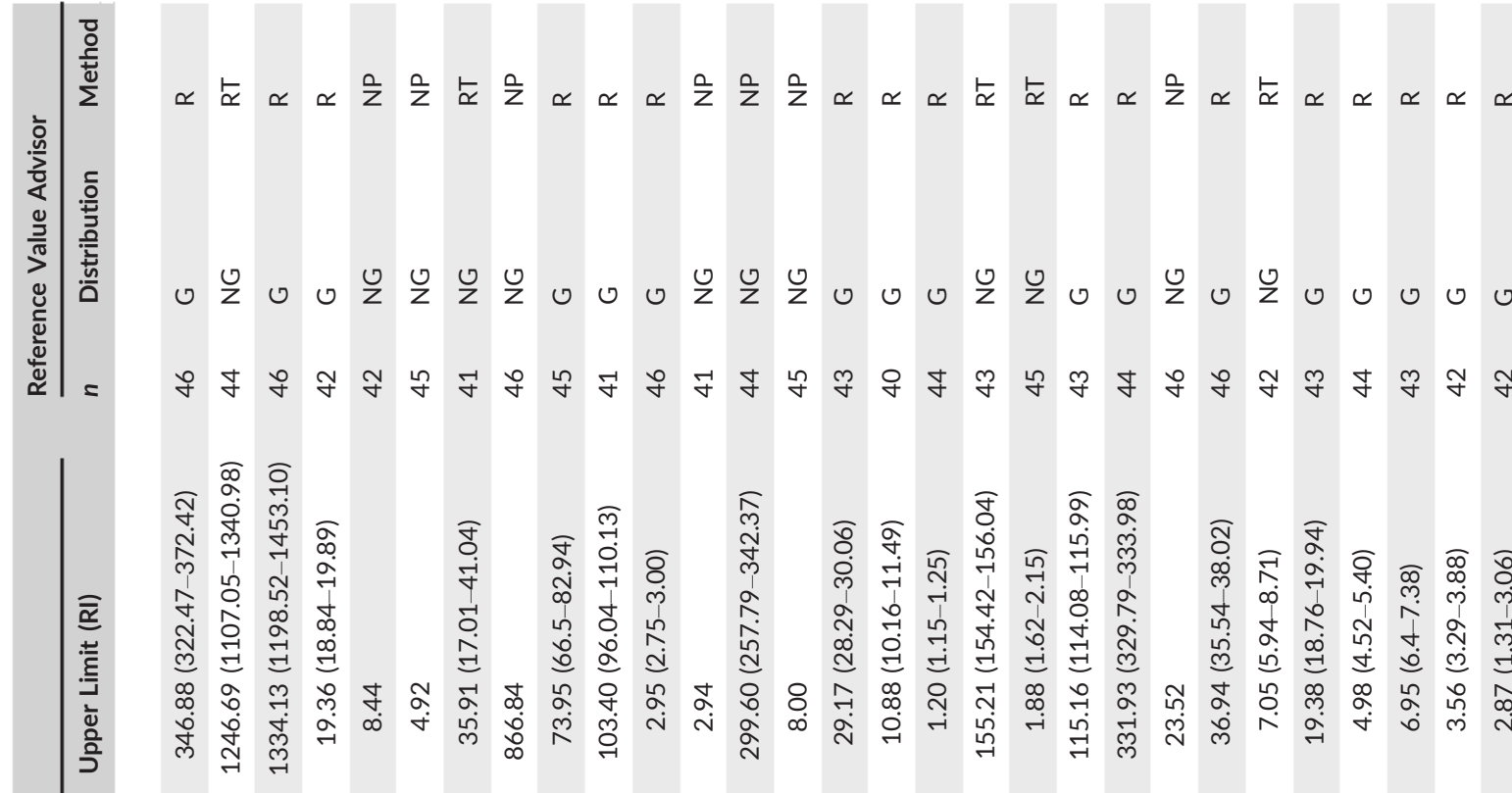

लु

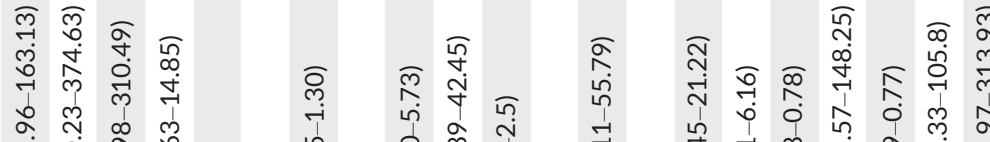

岱

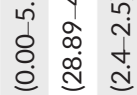

in

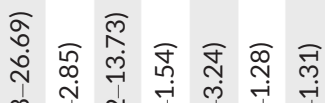

ปี

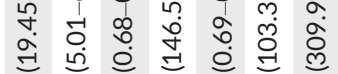

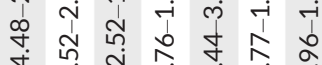

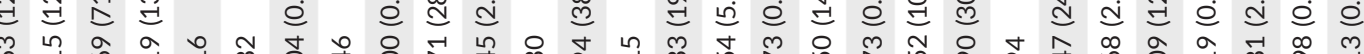
离

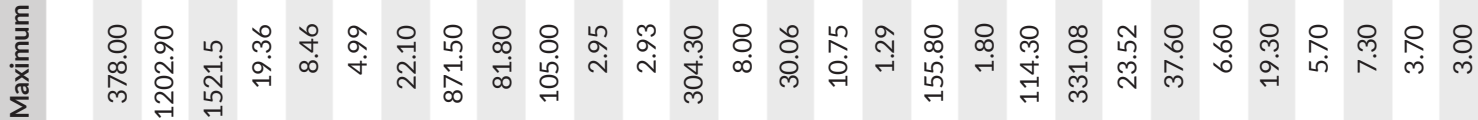

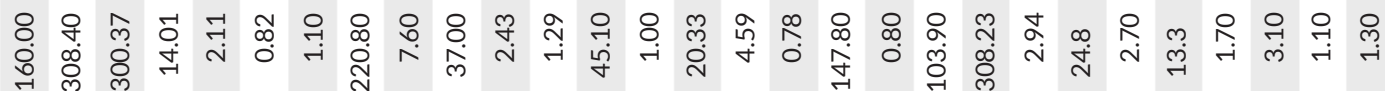

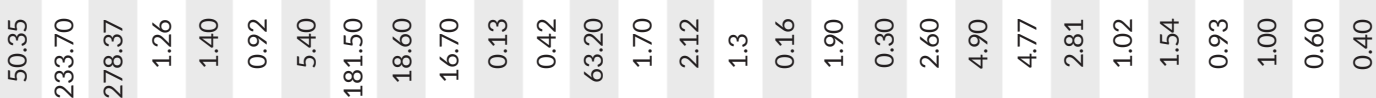

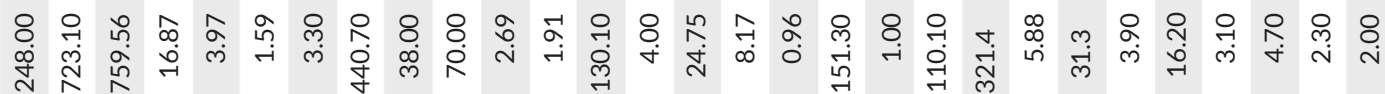

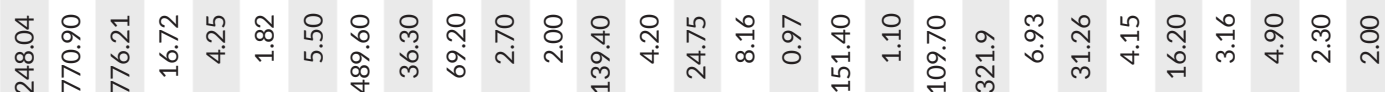

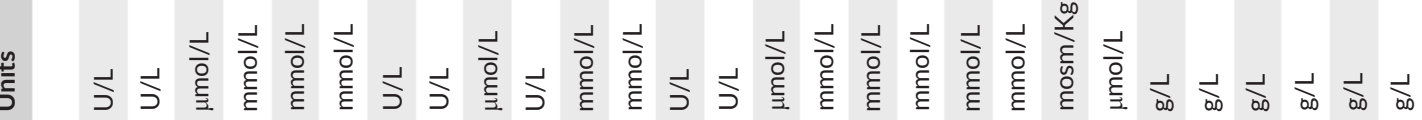

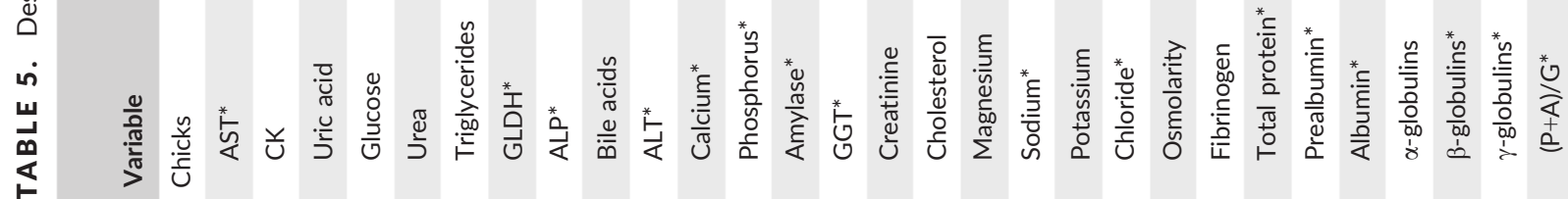




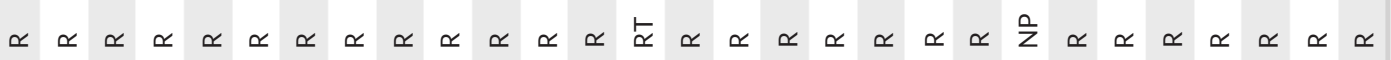

$=$

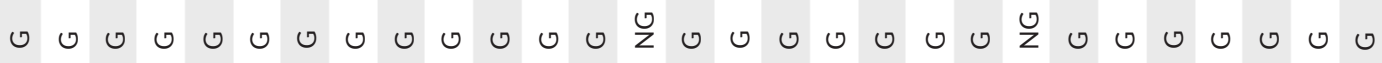
กิ

लำ

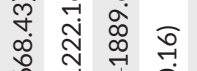

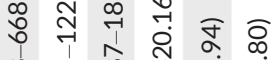

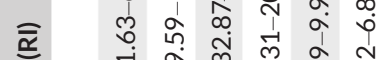

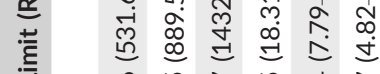

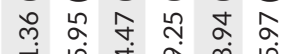

究

สิ๊

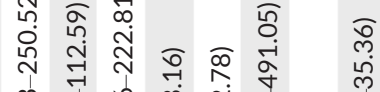

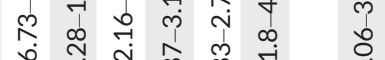

今ે

वे

तें

苍

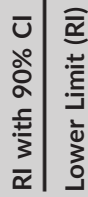

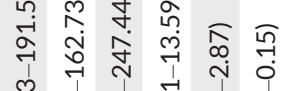

^

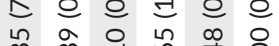

崩

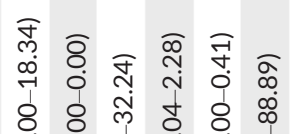

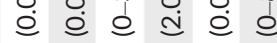

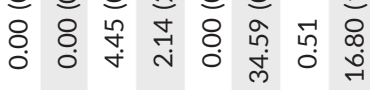

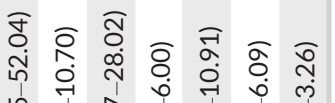

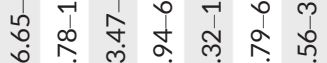

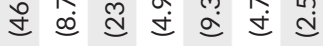

亲

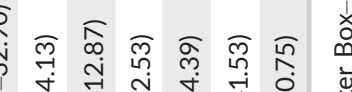

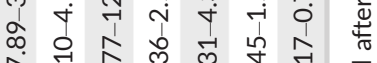

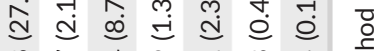

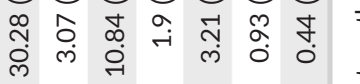

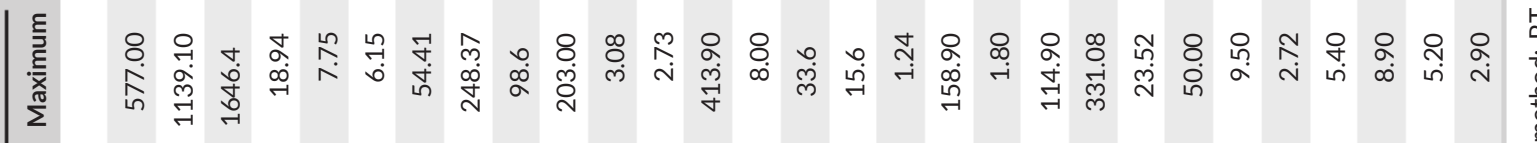

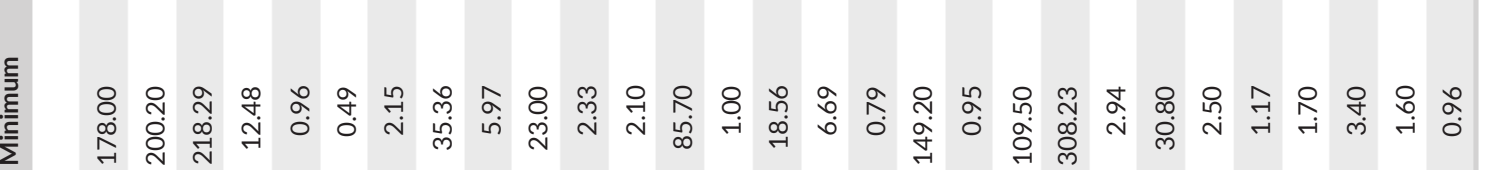

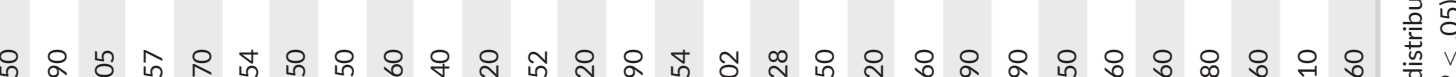

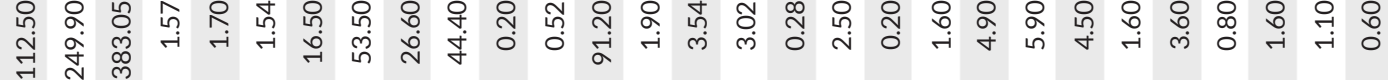

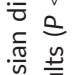

○

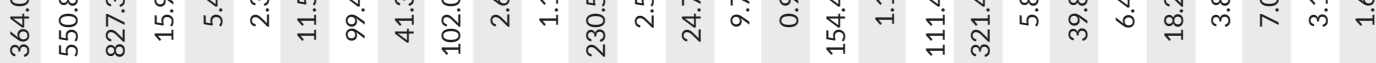

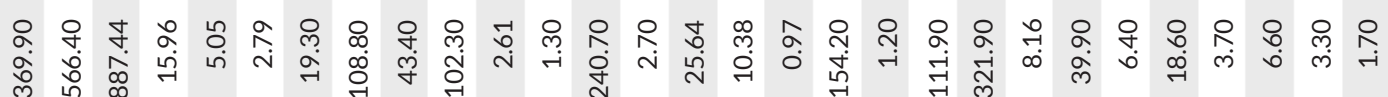

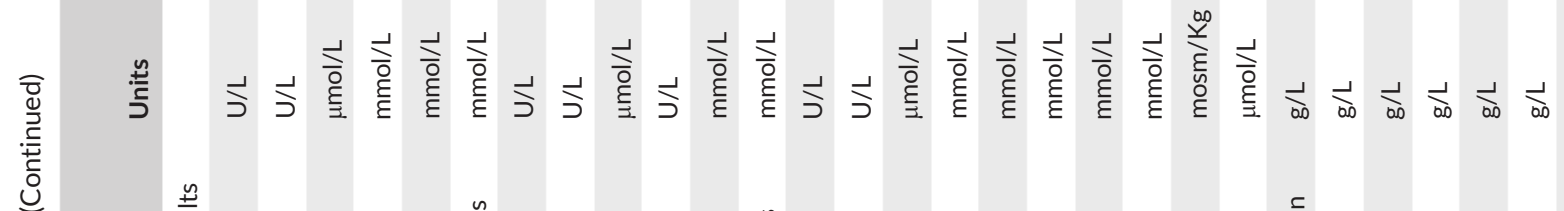

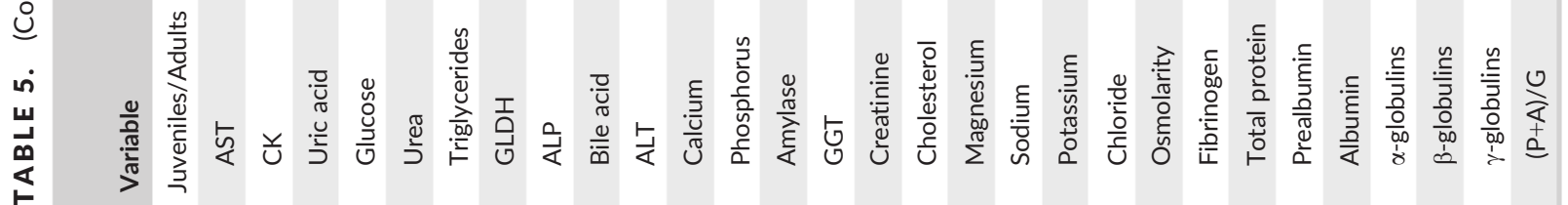


TABLE 6. Blood biochemistry analyte reference values for healthy Long-eared owl chicks.

\begin{tabular}{|c|c|c|}
\hline AST & $\mathrm{U} / \mathrm{L}$ & $194.00,251.00,222.00,304.00,292.00,225.00,248.00$ \\
\hline CK & $\mathrm{U} / \mathrm{L}$ & $273.90,206.50,243.70,257.00,147.60,397.40,259.10$ \\
\hline Uric acid & $\mu \mathrm{mol} / \mathrm{L}$ & $258.10,283.1,0520.50,402.10,607.90,861.80,978.50,648.00$ \\
\hline Glucose & $\mathrm{mmol} / \mathrm{L}$ & $16.40,16.00,22.40,18.70,18.70,17.80,19.90,13.80$ \\
\hline Urea & $\mathrm{mmol} / \mathrm{L}$ & $2.10,4.10,3.60,3.60,4.30,4.90,5.50$ \\
\hline Triglycerides & $\mathrm{mmol} / \mathrm{L}$ & $0.70,1.20,1.00,1.05,1.28,0.71,1.19$ \\
\hline ALT & $\mathrm{U} / \mathrm{L}$ & $112.00,58.00,97.00,81.00,86.00,60.00,80.00,65.00$ \\
\hline Calcium & $\mathrm{mmol} / \mathrm{L}$ & $2.60,2.70,2.40,2.60,2.70,2.60,2.60,2.80$ \\
\hline Phosphorus & $\mathrm{mmol} / \mathrm{L}$ & $1.60,2.80,2.10,2.20,2.30,2.70,2.30,2.30$ \\
\hline Amylase & $\mathrm{U} / \mathrm{L}$ & $111.10,75.60,109.80,101.00,75.50,87.30$ \\
\hline GGT & $U / L$ & $2.00,3.00,3.00,4.00,4.00,2.00,1.00$ \\
\hline Cholesterol & $\mathrm{mmol} / \mathrm{L}$ & $7.10,8.70$ \\
\hline Magnesium & $\mathrm{mmol} / \mathrm{L}$ & $1.10,1.20$ \\
\hline Sodium & $\mathrm{mmol} / \mathrm{L}$ & $154.80,171.10$ \\
\hline Potassium & $\mathrm{mmol} / \mathrm{L}$ & $1.60,2.80$ \\
\hline Chloride & $\mathrm{mmol} / \mathrm{L}$ & $113.50,137.30$ \\
\hline Osmolarity & $\mathrm{mosm} / \mathrm{Kg}$ & 246.97 \\
\hline Fibrinogen & $\mu \mathrm{mol} / \mathrm{L}$ & $11.70,8.80,11.80,11.70,14.70,11.80,11.80,5.90,2.94,5.88$ \\
\hline Total Protein & $\mathrm{g} / \mathrm{L}$ & $30.10,31.40,32.60,32.50,30.50,34.80$ \\
\hline Prealbumin & $\mathrm{g} / \mathrm{L}$ & $2.20,2.00,2.40,3.20,2.70,4.20,7.30$ \\
\hline Albumin & $\mathrm{g} / \mathrm{L}$ & $13.50,14.10,13.90,16.60,16.50,15.20,13.00$ \\
\hline$\alpha$-globulins & $\mathrm{g} / \mathrm{L}$ & $1.80,2.30,3.00,2.40,2.70,3.30$ \\
\hline$(P+A) / G$ & & $1.40,1.10,1.10,1.60,1.40,1.80,1.40$ \\
\hline
\end{tabular}

free of hemolysis and lipemia. Although no plasma samples had to be excluded due to this cause, the volumes of some plasma specimens were insufficient for standard statistical RI for some biochemical variables (be produced during strenuos exercise as for instance in animals are under a constraint).

Descriptive statistics (mean, median, standard deviation, minimum, and maximum) and Rls with the $90 \% \mathrm{Cl}$ for the upper and lower limits of cholesterol, osmolality, and albumin in the Iberian Strigiformes are presented in Table 2).

Descriptive statistics (mean, median, standard deviation, minimum, and maximum) and Rls with a $90 \% \mathrm{Cl}$ of upper and lower limits of Tawny owl, Little owl, Scops owl, and Barn owl by age (oneway ANOVA; $P<.01$ ) are shown in Tables 3-7.

Most statistically significant differences were seen between the chicks and the juvenile/adults and while no differences were observed between juveniles and adults. Therefore, Rls were determined for juveniles and adults as one combined group for any analyte, which resulted in the grouping of values from these 2 groups into one category. Figures 1-5 show the mean with the Cls (95\%) of ALP, $\mathrm{P}, \mathrm{Ca}, \mathrm{Na}$, and $\gamma$-globulins between age groups in the 5 species of Iberian Strigiformes species investigated here. Of all analytes measured, only ALP and phosphorus showed age-related changes in all owl species and values decreased with age. In the Strigidae family, sodium and the $\gamma$-globulins increased, and calcium decreased with age. In the Tawny owl, concentrations of triglycerides, creatinine, magnesium, and total protein were lower in chicks, while that GGT activity and potassium concentration were higher in chicks than in juveniles and adults. In the Little owl, CK activity, and concentrations in prealbumin, and potassium were higher in chicks. In the Scops owl, activities of AST, ALT, amylase, and GLDH activities, and concentrations of chloride, total protein, prealbumin, albumin, and $\beta$-globulins were lower in chicks, and GGT activity was higher in chicks than in juveniles and adults.

Finally, in the Barn owl, only AST activity was lower in chicks than in juveniles and adults.

\section{DISCUSSION}

Studies of avian plasma chemistry are of value to ecologists and veterinarians, especially for the management of threatened species.

To our knowledge, this is the first study to report blood chemistry Rls for the Little owl and the Scops owl, and biochemical Rls 


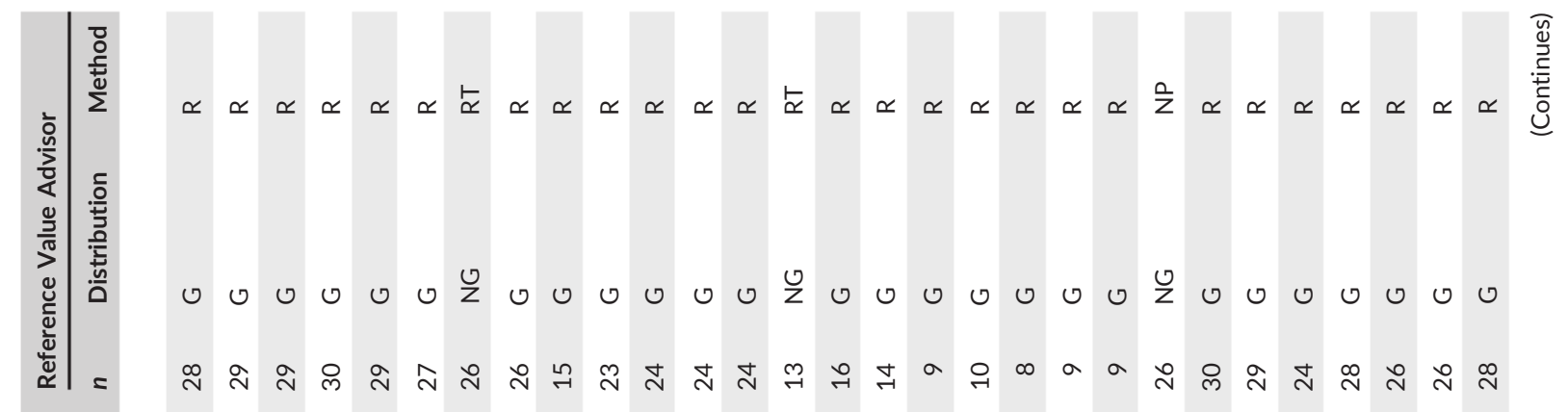

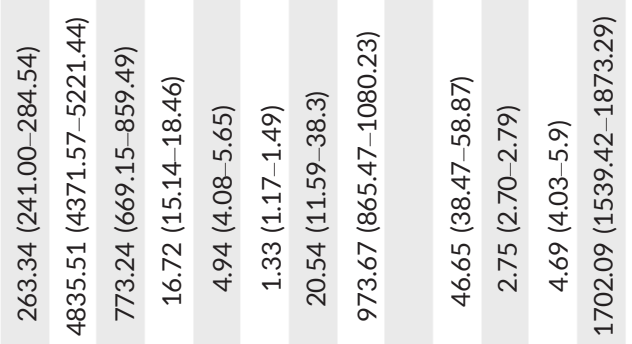

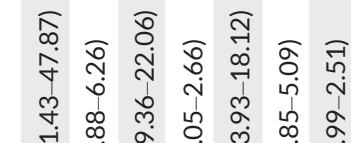

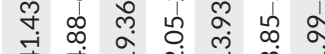

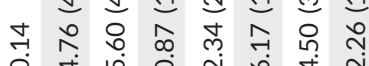

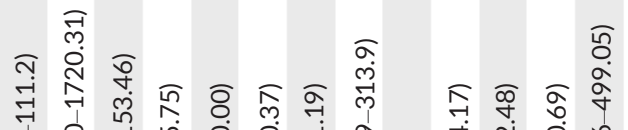

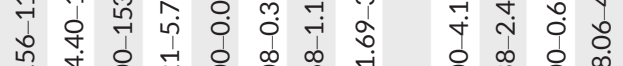

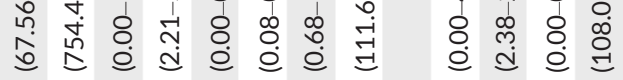

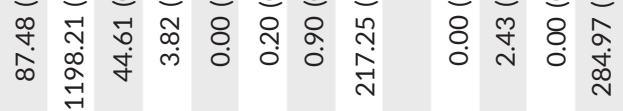

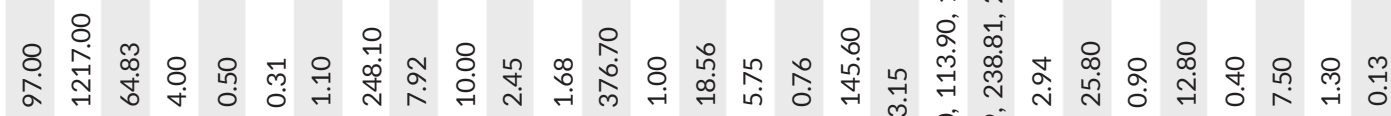

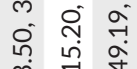

\section{0}

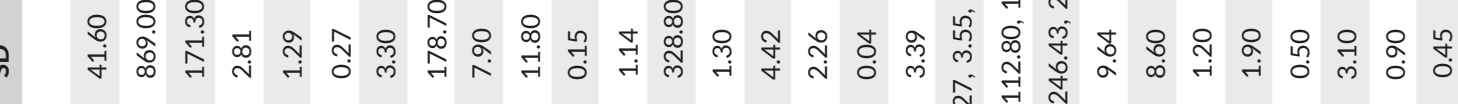
ले हैं

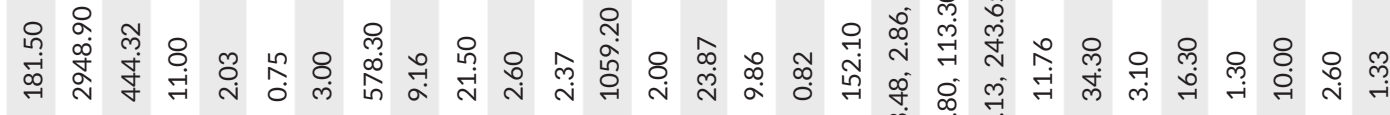
隽

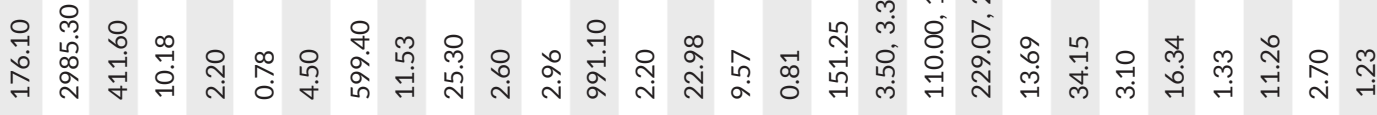

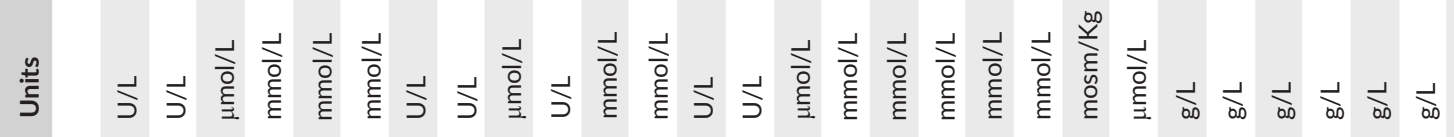

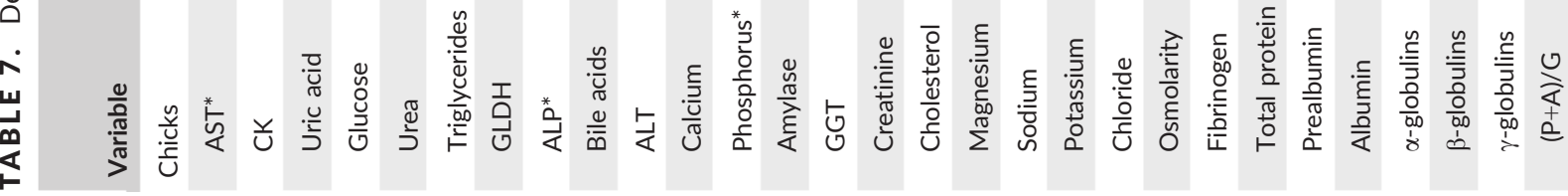




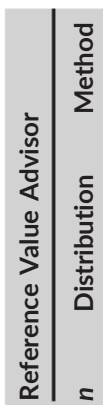

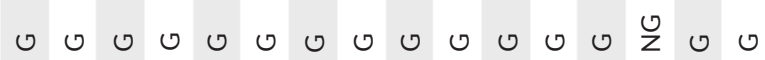

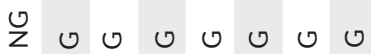

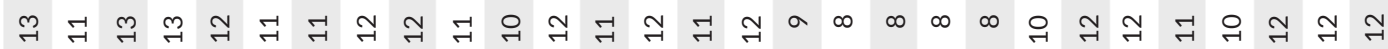

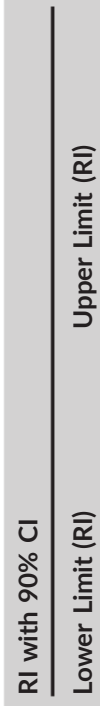

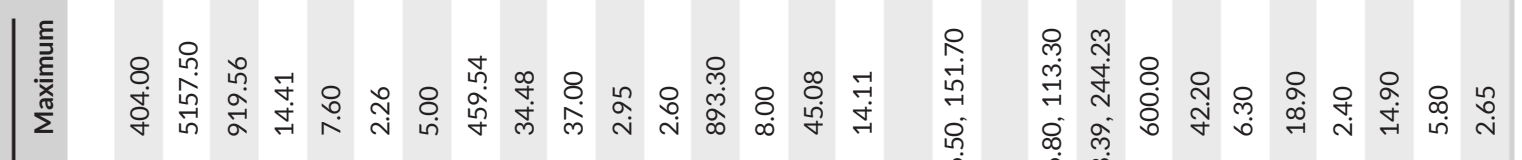
ह⿸丆口 นึ่

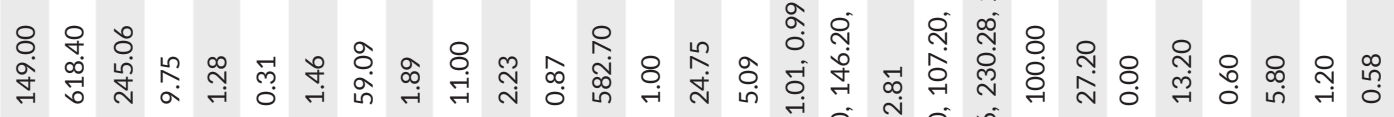

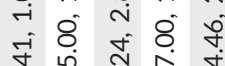
Q 0.0 m

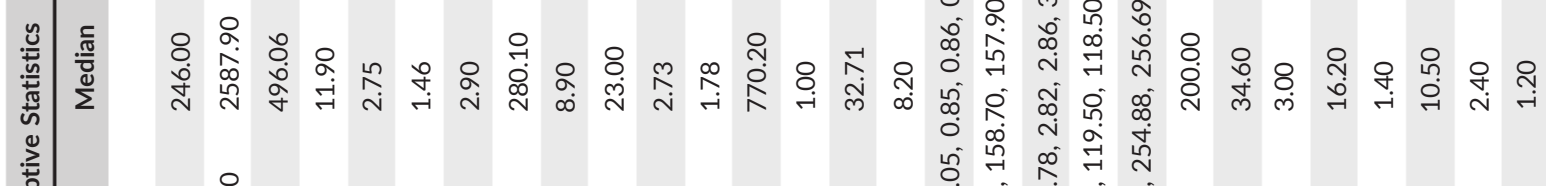

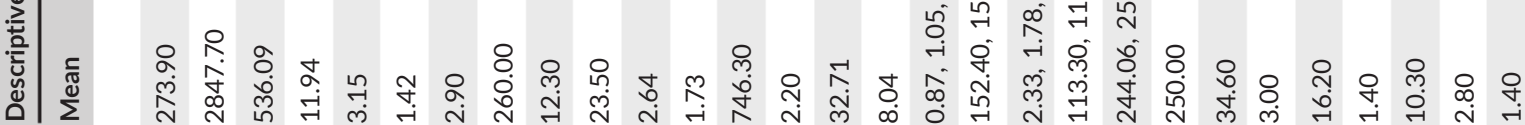
定. 政 言旁咅

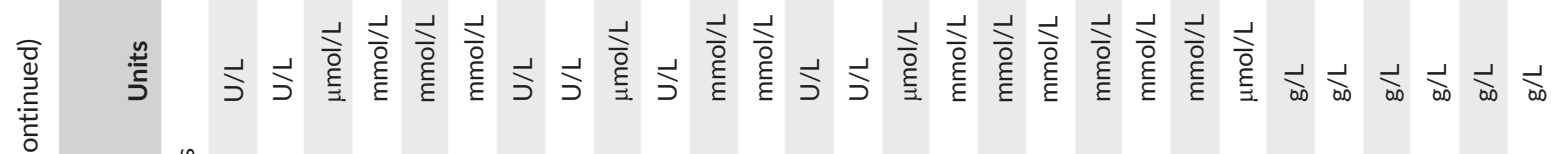




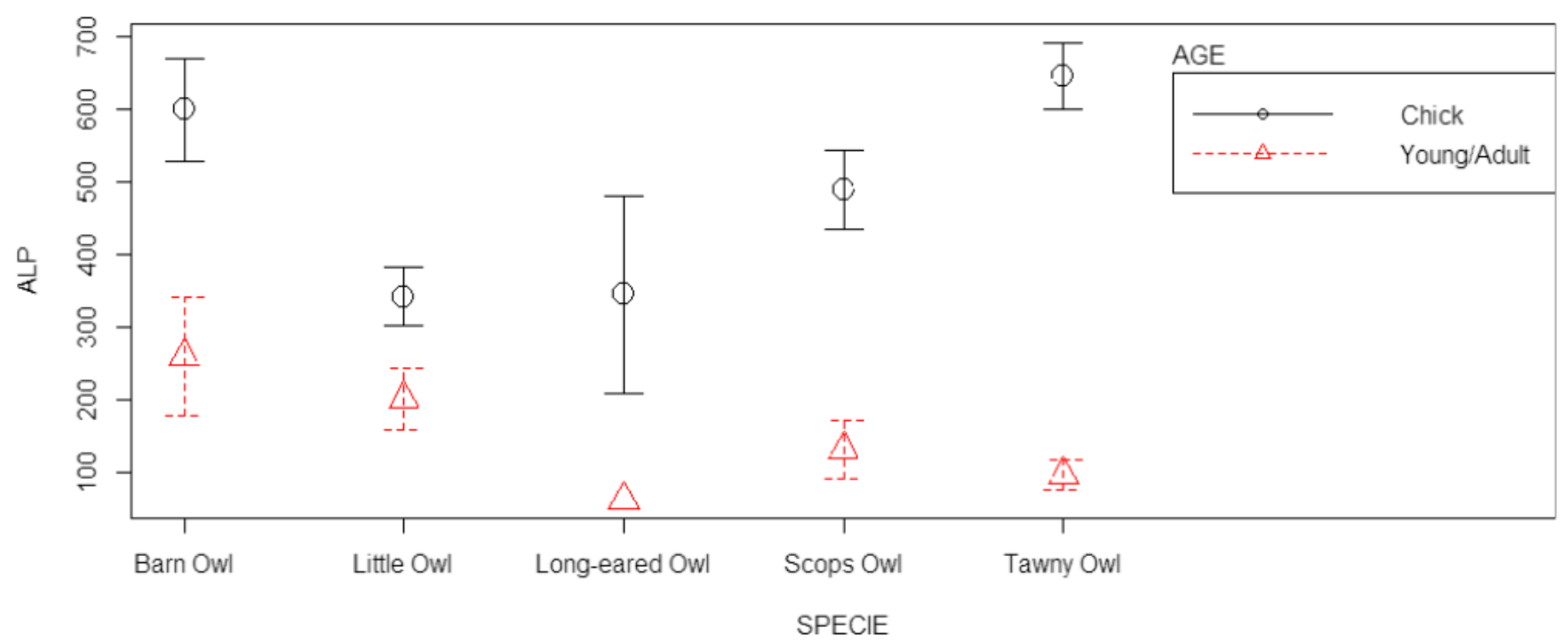

FIGURE 1. Mean with Cls $95 \%$ of ALP by age and Strigiformes species.

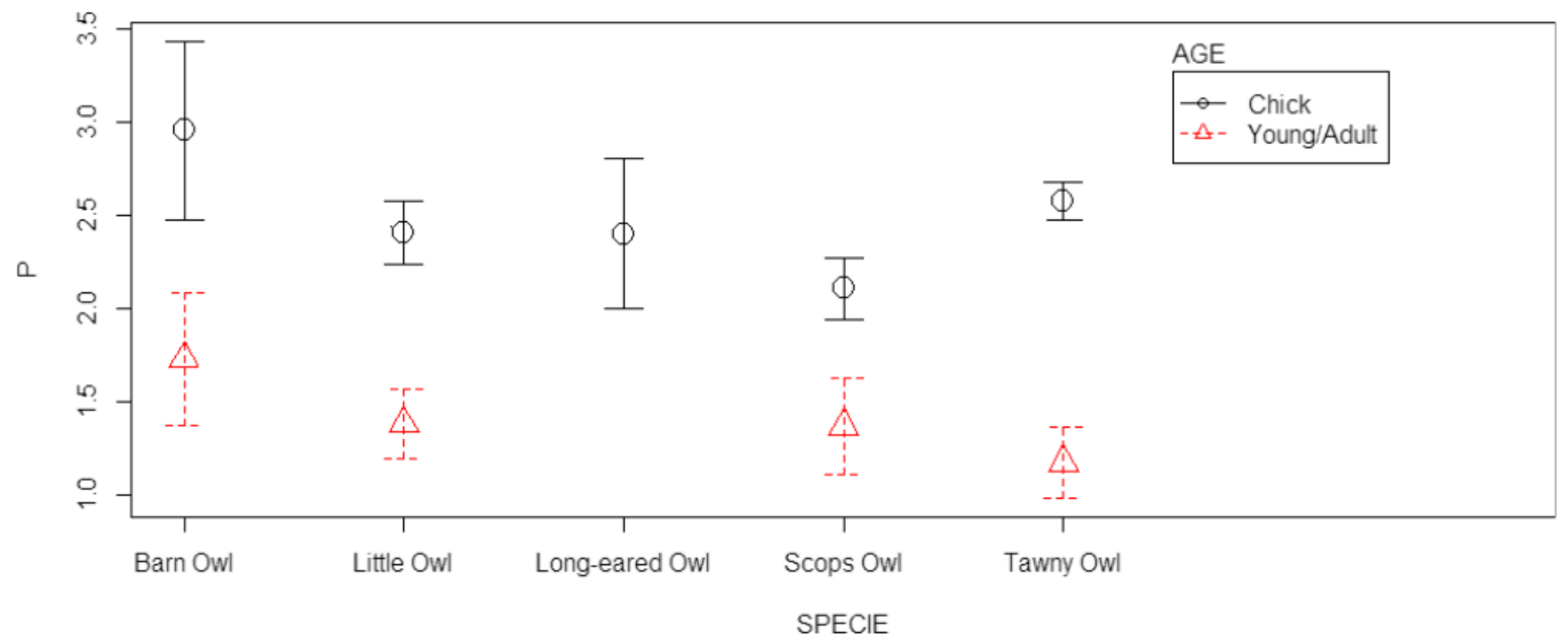

FIGURE 2. Mean with $\mathrm{Cls} 95 \%$ of $\mathrm{P}$ by age and Strigiformes species.

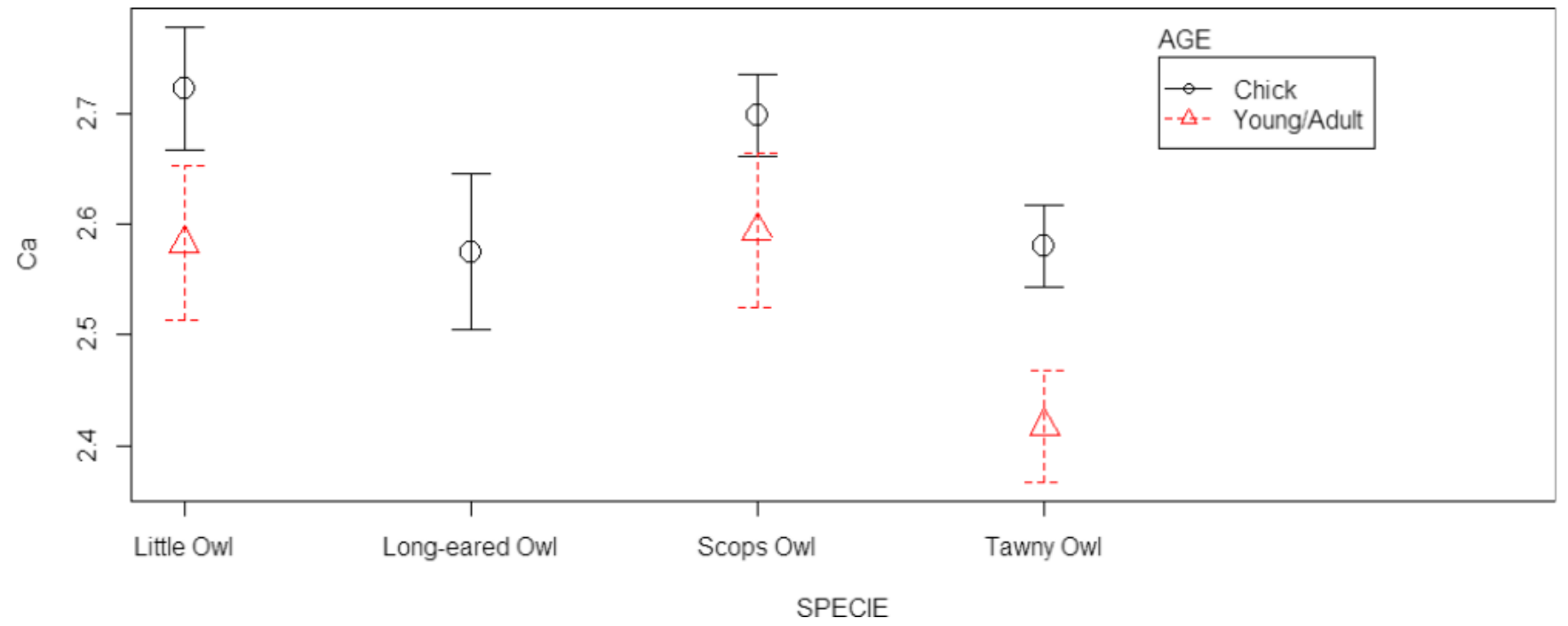

FIGURE 3. Mean with Cls $95 \%$ of $\mathrm{Ca}$ by age and Strigiformes species. 


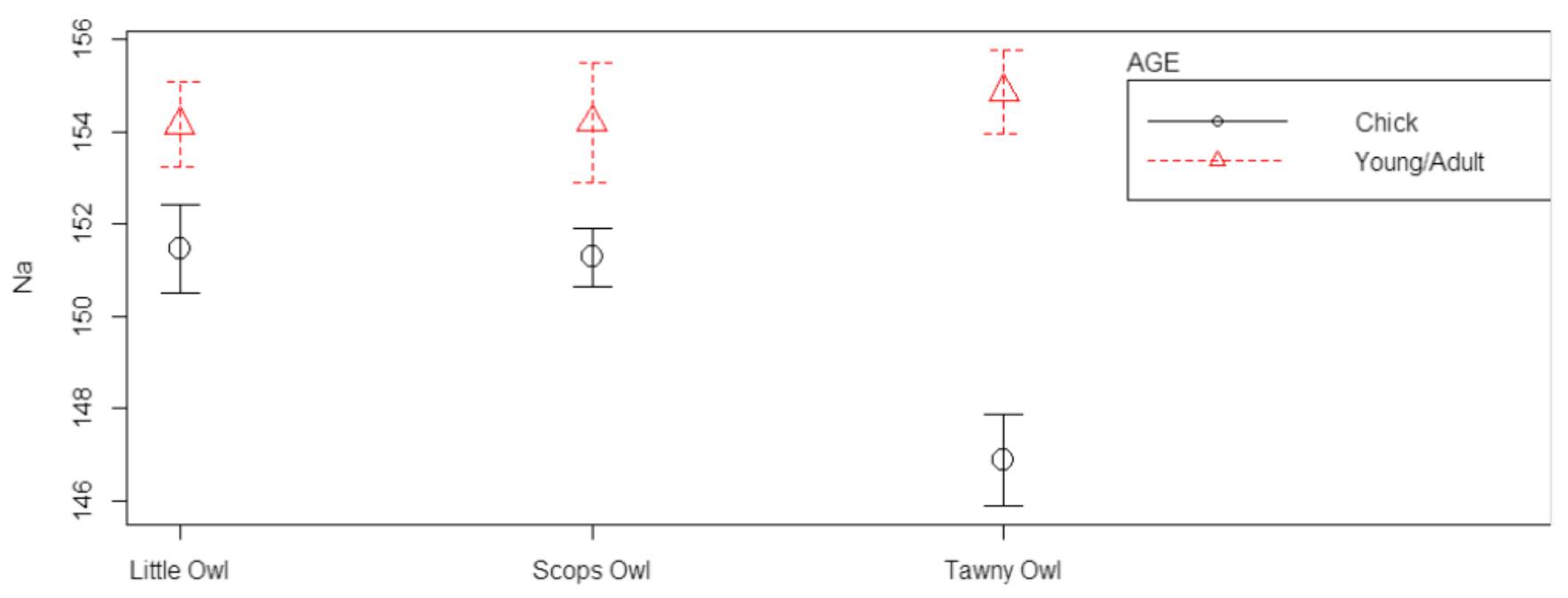

SPECIE

FIGURE 4. Mean with $\mathrm{Cls} 95 \%$ of $\mathrm{Na}$ by age and Strigiformes species.

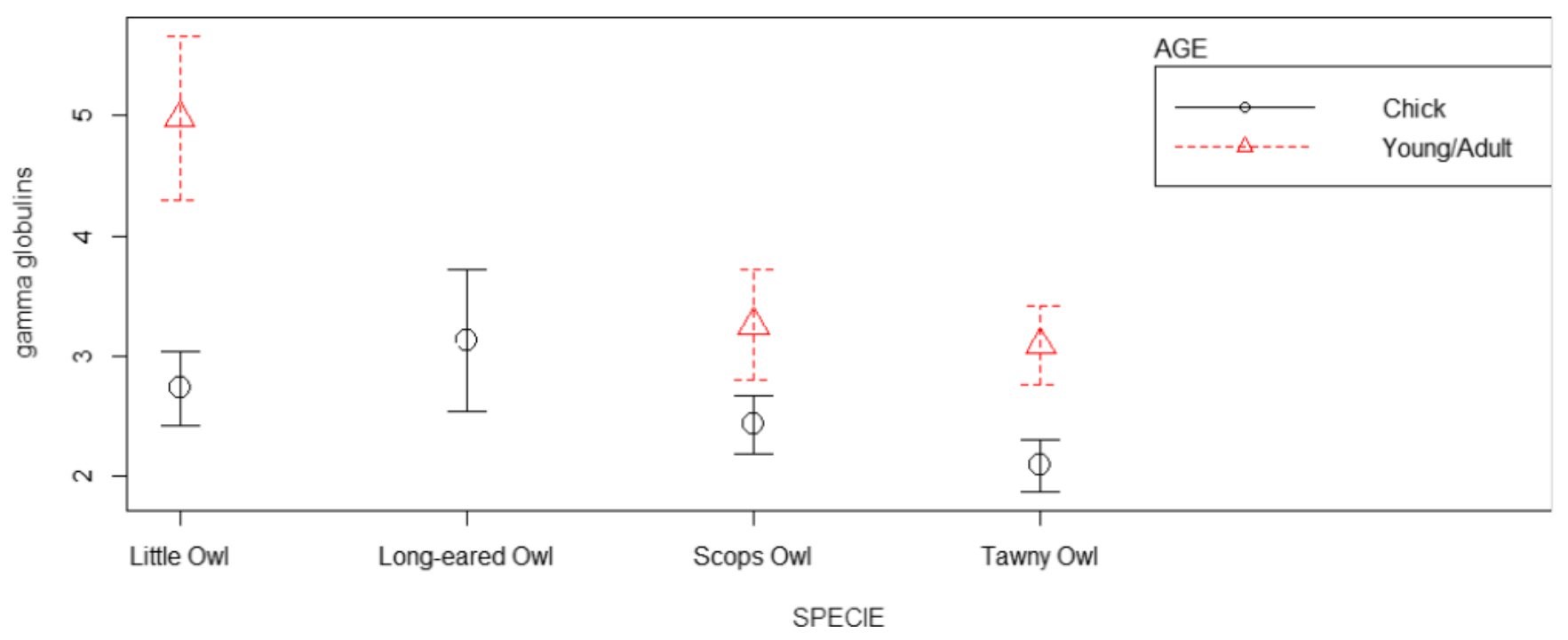

FIGURE 5. Mean with Cls $95 \%$ of $\gamma$-globulins by age and Strigiformes species.

in different age groups of Tawny owl, Little owl, Scops owl, Longeared owl, and Barn owl with representative numbers in each group.

In general, the biochemical analyte levels of the studied species were within the range of variation documented for other avian species, but CK, AST, and GLDH activities, and uric acid concentrations were slightly higher. ${ }^{19}$ As previously established, CK activity usually originates from skeletal muscle, heart muscle, and brain tissue, hence is considered a diagnostic indicator of muscle cell disruption, which can be produced during strenuos exercise as for instance in animals are under a constraint also during strenuous exercise as for instance in animals under constraint. ${ }^{20}$

A variety of tissues, predominately liver, and muscle, contain high AST concentrations. ${ }^{3}$ Most avian species have AST activities at the upper limit of $230 \mathrm{U} / \mathrm{L}$, and the AST values in the Strigiformes of this study were higher. Stress and exercise cause increased CK and
AST activities in blood, and these are higher during the capture and handling of wild species. ${ }^{21}$ These procedures are inevitable in the process of blood sample extraction, and therefore this change in the blood values of CK and AST should be considered and included in the RI. ${ }^{22}$

GLDH is an enzyme located in hepatocytes and poorly investigated in raptors. Although values above $10 \mathrm{UI} / \mathrm{L}$ have been associated with severe hepatic injury ${ }^{23}$, our results and those recently obtained $^{9}$ show that healthy Strigiformes have high activities. Since we have not associated these high concentrations with liver alterations or disease, the significance is unknown.

The Rl of blood uric acid concentration in raptors is approximately $50 \%$ higher than in granivorous and frugivorous avian species. ${ }^{24}$ Our results agree with the assertion that blood levels are closely related to diet composition and that uric acid increases with increasing proportions of ingested protein. ${ }^{25}$ 
Interspecies variability in blood biochemistry values has been documented in other phylogenetic groups of birds. ${ }^{26}$ In our study, statistically significant differences were found among the 5 species investigated, which confirms the following rule: interpretation of blood biochemistry results in particular owl species can only be performed in comparison with species-specific Rls. ${ }^{8}$ Cholesterol concentrations did not show interspecies variability, which may be due to the uniform diet ingested by all animals given the demonstrated relationship between this metabolite and food composition. ${ }^{25}$

Albumin plays a critical role in controlling oncotic pressure in biologic systems. The fluid volume of the intracellular and extracellular compartment is determined by the relative osmolality. ${ }^{26}$ The fact that the cholesterol and albumin did not show interspecies variability could be due to a uniform mechanism for maintaining water balance within the Strigiforme family living in the same environmental conditions. This hypothesis is supported by the results of the comparative study on 4 genera of parrots, where differences between all biochemical blood analyte values except plasma osmolality were reported. ${ }^{27}$

Trends related to phylogeny were identified. The Barn owl had lower plasma glucose concentrations and higher CK activities than the Strigidae family, similar to another study. ${ }^{9}$ The reasons for these differences are unknown, but we think they could be due to physiologic differences in insulin and glucagon metabolism or in variations of responses to stress among species.

Variability in biochemical analytes of the same species of birds, but between different studies has been well documented. ${ }^{28}$ We believe that the study differences are due to 2 aspects of the preanalytic phase: the genetic and physiological differences between the populations sampled, and the handling and processing of samples using different laboratory techniques. ${ }^{17}$ This variability supports the assumption that RI generation should be a systematic process, with stringent definition of the reference population, standard operating procedures in sample handling, method standardization, analytic variation, equipment used, and the use of valid statistical methods. ${ }^{18}$

It is widely confirmed in the literature that the age of the bird influences its blood chemistry. Comparisons between age groups obtained in this study are similar to findings in captive California condors (Gymnogyps californianus), where differences were primarily concentrated in condors $<6$ months of age, ${ }^{29}$ and chicks of the Egyptian vulture (Neophron percnopterus ginginianus). ${ }^{30}$ Therefore, chicks have to be considered a separate group from juveniles and adults.

Of all the analytes measured, only ALP, calcium, and phosphorus showed differences between age groups in the species compared. These findings are comparable to those observed in nestlings of Egyptian vultures, ${ }^{30}$ free-living Spoonbills (Platalea leucorodia), ${ }^{31}$ Pigeon guillemots (Cepphus columba) ${ }^{32}$, Black vultures (Coragyps atratus), ${ }^{33}$ and free-living European starlings (Sturnus vulgaris). ${ }^{34}$

Alkaline phosphatase is an enzyme closely associated with calcium and phosphorus metabolism, chondrogenic and osteoblastic activities in skeletal growth, and secondary ossification in birds, ${ }^{11}$ under hormonal control. We observed higher ALP activities in chicks than in juveniles and adults. This agrees with findings previously reported in Pigeon guillemots ${ }^{32}$ and Black vultures. ${ }^{33}$ The best evidence of lower ALP activity in adult birds was provided by a study of Spanish imperial eagles, where regression curves of ALP in nestlings, juveniles, and adults demonstrated an age-related decrease in this enzyme activity. ${ }^{35}$

Calcium and phosphorus levels may reflect intense osteoblastic activity and bone metabolism required for somatic growth in nestlings. ${ }^{11}$ In pigeon guillemots, calcium and phosphorus concentration in plasma were substantially higher in nestlings when compared with adults. ${ }^{32}$ Similarly, the nestlings of black kites (Milvus migrans) and red kites (Milvus milvus) ${ }^{11}$ and Spanish imperial eagles (Aquila adalberti) ${ }^{36}$ showed higher levels of calcium and phosphorus than adult birds. Thus, the higher activity of ALP, and concentrations of calcium and phosphorus observed in chicks of these owl species may be associated with bone mineralization and osteoblast activity during the growth period.

In the Strigidae family (Tawny owl, Little owl, and Scops owl), the concentration of sodium and plasma $\gamma$-globulins also significantly increased with age. The lower concentration of plasma $\gamma$-globulins in chicks has been observed in free-living European starlings. ${ }^{37}$ In birds the consumption of passive maternal antibodies usually occurs by 28 days of age. This age is approximately the same as that of the group of chicks in our study, and the observed increase in juvenile and adult concentrations might coincide with the development of active immunity. ${ }^{38-40}$

This is the first study to establish biochemical Rls determined using the same and specified methods according to age groups in the Tawny owl, Little owl, Scops owl, Long-eared owl, and Barn owl. Statistically significant differences were found between means of some analytes in the 5 owl species investigated, while the RI of cholesterol, osmolality, and albumin did not show interspecies variability. Most differences between age groups were between the chicks compared with the juvenile/adults, so this young age cohort necessitates an independent RI. Finally, no differences were seen in biochemical analytes between juveniles and adults, which allowed grouping a single category.

We recommend that these results be used to guide the analysis of blood biochemistry of the species investigated, especially for populations of animals housed in similar environmental conditions at centers of wildlife recovery.

\section{ACKNOWLEDGMENTS}

The authors thank Elena Obón, and Torreferrusa and Vallcalent wildlife recovery centers for technical assistance during the conduct of this investigation.

\section{DISCLOSURE}

The authors have indicated that they have no affiliations or financial involvement with any organization or entity with a financial interest in, or in financial competition with, the subject matter or materials discussed in this article. 


\section{REFERENCES}

1. Molina-López RA, Casal J, Darwich L. Specie-specific outcomes of wild Raptors attended at a wildlife Rehabilitation centre in Catalonia (1997-2005). Am J Anim Vet Sci. 2014;9:19-27.

2. Low M, Eason D, Elliott G, Mclnnes K, Paul-Murphy J. Hematologic and biochemical references ranges for the Kakapo (Strigops habroptilus): generation and interpretation in a field-based wildlife recovery program. J Avian Med Surg. 2006;20:80-88.

3. Franson JC, Murray HC, Bunck C. Enzyme activities in plasma, kidney, liver, and muscle of five avian species. J Wildl Dis. 1985;21: 33-39.

4. Tatum L, Zaias J, Mealey BK, Cray C, Bossart GD. Protein electrophoresis as a diagnostic tool in raptor medicine. J Zoo Wildl Med. 2000;31:497-502

5. Spagnolo V, Crippa V, Marzia A, Alberti I, Sartorelli P. Hematologic, biochemical, and protein electrophoretic values in captive tawny owls (Strix aluco). Vet Clin Pathol. 2008;37:225-228.

6. Chan FT, Lin PI, Chang GR, Wang HC, Hsu TH. Hematocrit and plasma chemistry values in adult collared scops owls (Otus lettia) and crested serpent eagles (Spilornis cheela hoya). J Vet Med Sci. 2012;74:893-898.

7. Chan FT, Lin PI, Chang GR, Wang HC, Hsu TH. Hematocrit and plasma chemistry values in adult collared scops owls (Otus lettia) and crested serpent eagles (Spilornis cheela hoya). J Vet Med Sci. 2012;74:893-898.

8. Ammersbach M, Beaufrere H, Gionet Rollick A, Tully T. Laboratory blood analysis in Strigiformes-Part II: plasma biochemistry reference intervals and agreement between Abaxis Vetscan V2 and the Roche Cobas c501. Vet Clin Pathol. 2015;44:128-140.

9. Szavo Z, Klein A, Jakab C. Hematologic and Plasma Biochemistry Reference Intervals of Healthy Adult Barn Owls (Tyto alba). Avian Dis. 2014;58:228-231.

10. Sturkie PD. Blood: physical characteristics, formed elements, hemoglobin and coagulation. In: Sturkie PD, ed. Avian Physiology. New York: Springer-Verlag; 1976:53-75.

11. Vinuela J, Ferrer M, Recio F. Age-related variations in plasma levels of alkaline phosphatase, calcium and inorganic phosphorus in chicks of two species of raptors. Comp Biochem Physiol A Physiol. 1991;99:4954.

12. van Wyk E, van der Bank $H$, Verdoon GH. Dynamics of haematology and blood biochemistry in free-living African whitebacked vulture (Pseudogyps africanus) nestlings. Comp Biochem Physiol A Physiol. 1998;120:495-508.

13. Haefele HJ, Sindor I, Evers DC, Hoyt DE, Pokras MA. Hematologic and physiologic references ranges for free-ranging adult and young common loons (Gavia immer). J Zoo Wildl Med. 2005;36:385-390.

14. Acierno MJ, Mitchell MA, Freeman DM, Schuster PJ, Sanchez-MigalIon D, Tully TN. Determination of plasma osmolality and agreement between measured and calculated values in healthy adult Hispaniolan Amazon parrots (Amazon ventralis). Am $J$ Vet Res. 2009;70:1151-1154.

15. Millar HT, Simpsom JG, Stalker AL. An evaluation of the heat precipitation method of fibrinogen estimation. J Clin Pathol. 1971;24:827-830.

16. Geffré A, Friedrichs K, Harr K, Concordet D, Trumel C, Braun J. Reference values: a review. Vet Clin Pathol. 2009;38:288-298.

17. Flatland $B$, Freeman KP, Friedrichs KR, et al. ASVCP quality assurances guidelines: control of general analytical factors in veterinary laboratories. Vet Clin Pathol. 2010;39:264-277.

18. Friedrichs RF, Harr KE, Freeman KP, et al. ASVCP reference interval guidelines: determination of de novo reference intervals in veterinary species and other related topics. Vet Clin Pathol. 2012;41:441-453.

19. Fudge AM. Laboratory reference ranges for selected avian, mammalian, and reptilian species. In: Fudge AM, ed. Laboratory medicine: Avian and Exotic Pets. Philadelphia, PA: WB Saunders; 2000:376-400.
20. Hortobagyi T, Denaham T. Variability in creatine kinase: methodological, exercise and clinically related factors. Int J Sports Med. 1989;10:69-80.

21. Bailey T, Wernery U, Howlett J, Naldo J, Samour J. Age-related plasma chemistry changes in Houbara and Kori Bustards in the United Arab Emirates. J Wildl Dis 1999;35:31-37.

22. Scope A, Filip T, Gabler C, Resch F. The influence of stress from transport and handling on hematologic and clinical chemistry blood parameters of racing pigeons (Columbia livia domestica). Avian Dis. 2002;46:224-229.

23. Campbell TW. Hematology of birds. In: Thrall MA, Weiser G, Allison RW, Campbell T, eds. Veterinary Hematology and Clinical Chemistry. 2nd ed. Oxford, UK: Wiley-Blackwell; 2012:238-276.

24. Gylstorff I, Grimm F. Vogelkrankheiten [Avian diseases]. Stuttgart Eugen Ulmer. 1987;133-146.

25. Lumeij JT. Avian Clinical Biochemistry. In: Kaneko JJ, Harvey JW, Bruss ML, eds. Clinical Biochemistry of Domestic Animals. 6th ed. San Diego, CA: Academic Press; 2008:839-872.

26. Kohn CW, DiBartola SP. Composition and distribution of body fluids in dogs and cats. In: Dibartola SP, ed. Fluid therapy in small animal practice. Philadelphia, PA: WB Saunders Co.; 2000:3-25.

27. Lumeij JT, Overduin LM. Plasma Chemistry references values in Psittaciformes. Avian Pathol. 1990;19:235-244.

28. Montesinos A, Sainz A, Pablos MV, Mazzcuccheill F, Tesouro MA. Hematological and plasma biochemical reference intervals in young white storks. J Wildl Dis 1997;33:405-412.

29. Dujowich M, Mazet JK, Zuba JR. Hematological and Biochemical references ranges for captive California condors (Gymnogyps californianus). J Zoo Wildl Med. 2005;36:590-597.

30. Dobado-Berrios PM, Tella JL, Ceballos O, Donazar JA. Effects of age and captivity on plasma chemistry values of Egyptian Vulture. Condor. 1998;100:719-725.

31. De le Court C, Aguilera E, Fernández R. Plasma chemistry values of free-living White spoonbills (Platalea leucorodia). Comp Biochem Physiol A Physiol 1995;112:137-141.

32. Seiser PE, Duffy LK, McGuire AD, Roby DD, Golet GH, Litzow MA. Comparison of pigeon guillemot (Cepphus columba), blood parameters from oiled and unoiled areas of Alaska eight years after the Exxon Valdez oil spill. Mar Pollut Bull. 2000;40:152-164.

33. Villegas A, Sánchez JM, Costillo E, Corbacho C. Blood chemistry and haematocrit of the Black Vulture (Aegypius monachus). Comp Biochem Physiol A Physiol. 2002;132:489-497.

34. Juráni $M$, Výboh $P$, Zeman $M$, Lamošová $D$, Kost'ál L, Blažžcek $P$. Post-hatching dynamics of plasma biochemistry in free-living European starlings (Sturnus vulgaris). Comp Biochem Physiol A Physiol. 2004;138:89-95.

35. Vinuela J, Ferrer M. Regulation of growth in red kites and imperial eagles. Wilson Bull. 1997;109:92-101.

36. Dobado-Berrios PM, Ferrer M. Age-related changes of plasma alkaline phosphatase and inorganic phosphorus and ossification of the cranial roof in the Spanish Imperial Eagle (Aquila adalberti). Physiol Zool. 1998;70:421-427.

37. Macari M, Gonzales E. Manejo da incubação [Incubation management]. Jaboticabal: Facta. 2003:537.

38. Conrado A, dos Anjos Lopes ST, Martins DB, et al. Eletroforese das proteínas plasmáticas em emas (Rhea americana) de diferentes faixas etárias [Electrophoresis of plasma proteins in rhea (Rhea americana) of different ages]. Ciencia Rural, Santa Maria. 2007;37:1033-1038.

39. Casado E, Balbontin J, Ferrer M. Plasma chemistry in booted Eagle (Hieraaetus pennatus) during breeding season. Comp Biochem Physiol A Physiol. 2002;131:233-241.

40. Baker JK. Identification Guide to European Non Passerines. UK: British Trust for Ornitology; 1993. 